\title{
Approximate Zero Modes for the Pauli Operator on a Region
}

\author{
Daniel M. Elton \\ Department of Mathematics and Statistics \\ Fylde College \\ Lancaster University \\ Lancaster LA1 4YF, United Kingdom \\ e-mail: d.m.elton@lancaster.ac.uk
}

May 4, 2015

\begin{abstract}
Let $\mathcal{P}_{\Omega, t A}$ denoted the Pauli operator on a bounded open region $\Omega \subset \mathbb{R}^{2}$ with Dirichlet boundary conditions and magnetic potential $A$ scaled by some $t>0$. Assume that the corresponding magnetic field $B=\operatorname{curl} A$ satisfies $B \in L \log L(\Omega) \cap C^{\alpha}\left(\Omega_{0}\right)$ where $\alpha>0$ and $\Omega_{0}$ is an open subset of $\Omega$ of full measure (note that, the Orlicz space $L \log L(\Omega)$ contains $L^{p}(\Omega)$ for any $p>1)$. Let $\mathrm{N}_{\Omega, t A}(\lambda)$ denote the corresponding eigenvalue counting function. We establish the strong field asymptotic formula

$$
\mathrm{N}_{\Omega, t A}(\lambda(t))=\frac{t}{2 \pi} \int_{\Omega}|B(x)| d x+o(t)
$$

as $t \rightarrow+\infty$, whenever $\lambda(t)=C e^{-c t^{\sigma}}$ for some $\sigma \in(0,1)$ and $c, C>0$. The corresponding eigenfunctions can be viewed as a localised version of the Aharonov-Casher zero modes for the Pauli operator on $\mathbb{R}^{2}$.
\end{abstract}

2010 Mathematics Subject Classification: 35P20, 35Q40, 35J47.

Keywords: Pauli operator, eigenvalue asymptotics, approximate zero modes.

\section{Introduction}

Let $\Omega \subset \mathbb{R}^{2}$ be a bounded open region and $A=\left(A_{1}, A_{2}\right) \in L_{\text {loc }}^{2}\left(\Omega, \mathbb{R}^{2}\right)$ a magnetic potential. The corresponding magnetic momentum operator is then $P_{A}=-i \nabla-A$, where $\nabla=\left(\nabla_{1}, \nabla_{2}\right)$ denotes the gradient operator on $\mathbb{R}^{2}$. We wish to consider the Pauli operator $\mathcal{P}_{\Omega, A}$ on $\Omega$ with magnetic potential $A$. For Dirichlet boundary conditions this can be defined as the non-negative operator $\mathcal{P}_{\Omega, A}$ associated to the closure of the form

$$
\mathbf{p}_{\Omega, A}(u)=\left\|P_{A,+} u_{+}\right\|^{2}+\left\|P_{A,-} u_{-}\right\|^{2}, \quad u=\left(\begin{array}{c}
u_{+} \\
u_{-}
\end{array}\right) \in C_{0}^{\infty}\left(\Omega, \mathbb{C}^{2}\right),
$$


where $P_{A, \pm}=P_{A, 1} \pm i P_{A, 2}$. Set $B=\operatorname{curl} A=\nabla_{1} A_{2}-\nabla_{2} A_{1}$, the magnetic field associated with the potential $A$ (initially defined as a distribution). A straightforward formal calculation leads to the Lichnerowicz formula

$$
\mathcal{P}_{\Omega, A}=\left(\begin{array}{cc}
H_{\Omega, A} & 0 \\
0 & H_{\Omega, A}
\end{array}\right)-\left(\begin{array}{cc}
B & 0 \\
0 & -B
\end{array}\right),
$$

where $H_{\Omega, A}=P_{A, 1}^{2}+P_{A, 2}^{2}$ is the magnetic Schrödinger operator. If we assume that $B$ belongs to the Orlicz space $L \log L(\Omega)$ then (2) can be rigorously justified and used to help show that $\mathcal{P}_{\Omega, A}$ has a compact resolvent and hence discrete spectrum (see Proposition 2.4). Enumerate the eigenvalues of $\mathcal{P}_{\Omega, A}$ (including multiplicities) as $0 \leq \lambda_{1}\left(\mathcal{P}_{\Omega, A}\right) \leq \lambda_{2}\left(\mathcal{P}_{\Omega, A}\right) \leq \ldots$, and introduce the corresponding counting function

$$
\mathrm{N}_{\Omega, A}(\lambda)=\#\left\{n \in \mathbb{N}: \lambda_{n}\left(\mathcal{P}_{\Omega, A}\right) \leq \lambda\right\}, \quad \lambda \in \mathbb{R} .
$$

We are interested in the behaviour of $\mathrm{N}_{\Omega, A}$ in the strong field regime. Fixing $A$ we consider $\mathrm{N}_{\Omega, t A}(\lambda(t))$ for the scaled potential $t A$ and $\lambda(t) \leq O(t)$ in the limit $t \rightarrow+\infty$. A simple rescaling shows that this is equivalent to the semi-classical regime.

When $\lambda(t)=O(t)$ the quantity $\mathrm{N}_{\Omega, t A}(\lambda(t))$ obeys a natural Weyl type asymptotics. To state this precisely introduce auxiliary functions $\nu^{-}$and $\nu^{+}$which are, respectively, the maximal lower and minimal upper semi-continuous extensions of

$$
\nu(b, \lambda)=\frac{|b|}{2 \pi} \#\{m \in \mathbb{Z}: 2|m b| \leq \lambda\}, \quad \lambda, b \in \mathbb{R}, b \neq 0, \lambda \notin 2|b| \mathbb{N}_{0} .
$$

Theorem 1.1. Suppose $B \in L \log L(\Omega) \cap C\left(\Omega_{0}\right)$ where $\Omega_{0} \subseteq \Omega$ is open and $\Omega \backslash \Omega_{0}$ has zero (Lebesgue) measure. If $\lambda(t)=\Lambda t+o(t)$ for some $\Lambda \in \mathbb{R}$ then

$$
\liminf _{t \rightarrow \infty} \frac{1}{t} \mathrm{~N}_{\Omega, t A}(\lambda(t)) \geq \int_{\Omega} \nu^{-}(B(x), \Lambda) d x
$$

and

$$
\limsup _{t \rightarrow \infty} \frac{1}{t} \mathrm{~N}_{\Omega, t A}(\lambda(t)) \leq \int_{\Omega} \nu^{+}(B(x), \Lambda) d x .
$$

Numerous results similar or related to Theorem 1.1 have been obtained. Some of the earliest work ([3], [27]) looked at spectral asymptotics for magnetic (Schrödinger) bottles. While these works focused on a different class of operators the ideas of [3] in particular form the basis of our approach to Theorem 1.1 (see also [28]). For magnetic Schrödinger operators on a region various two term spectral asymptotic questions have been considered in both the Dirichlet and Neumann cases (see [14], [11], [4], [12] and references therein); whilst giving more precise details, these results also require greater regularity (and other conditions) on $\Omega$ and $B$.

In another direction, various authors have considered bound states of the Pauli operator with an additional electric potential. The presence of the latter distinguishes the strong field and semi-classical regimes, leading to multi-parameter problems. The semi-classical behaviour of sums of negative eigenvalues of the form 
$\sum_{n}\left|\lambda_{n}\right|^{\gamma}, \gamma>0$ was considered in [20], [8], [25] and [9] for example. These works all rely on a priori bounds on the eigenvalue sums which have the correct order in the parameters; typically Lieb-Thirring type inequalities have been developed for this purpose. However eigenvalue counting corresponds to the case $\gamma=0$ (also known as the CLR inequality) and is always excluded in dimension 2.

The asymptotic bounds in Theorem 1.1 remain finite provided $B \in L^{1}(\Omega)$. We use the slightly stronger condition $B \in L \log L(\Omega)$ to obtain a priori bounds on $\mathrm{N}_{\Omega, t A}(\lambda(t))$ (covering the lack of a suitable Lieb-Thirring/CLR inequality); in turn these bounds are derived from estimates in [26] which don't extend to cover the $L^{1}$ case. The continuity condition $B \in C\left(\Omega_{0}\right)$ relates to our method for approximating $B$ locally by a constant field. While it is likely that at least the latter condition can be relaxed the optimal regularity condition for $B$ remains unclear.

The asymptotic lower and upper bounds given by Theorem 1.1 differ if the set

$$
\left\{x \in \Omega: 2 m|B(x)|=\Lambda \text { for some } m \in \mathbb{N}_{0}\right\}
$$

has non-zero measure. When $\Lambda \neq 0$ this is a non-generic situation for variable fields. On the other hand, when $\lambda(t)=o(t)$ (4) is the whole of $\Omega$ for any $B$; the lower bound in Theorem 1.1 then reduces to 0 while the upper bound becomes

$$
\limsup _{t \rightarrow \infty} \frac{1}{t} \mathrm{~N}_{\Omega, t A}(\lambda(t)) \leq \Phi_{\Omega}(|B|),
$$

where

$$
\Phi_{\Omega}(b)=\frac{1}{2 \pi} \int_{\Omega} b(x) d x
$$

is the flux of a magnetic field $b$ on $\Omega$ (see the end of Section 2 for some further details). It transpires that the upper bound gives the correct asymptotics for even sub-exponentially decaying $\lambda(t)$. Our main result is the following (in which $C^{\alpha}$ is used to denote the space of Hölder continuous functions).

Theorem 1.2. Suppose $B \in L \log L(\Omega) \cap C^{\alpha}\left(\Omega_{0}\right)$ where $\alpha>0, \Omega_{0} \subseteq \Omega$ is open and $\Omega \backslash \Omega_{0}$ has zero (Lebesgue) measure. If $\lambda(t) \geq C e^{-c t^{\sigma}}$ for some constants $\sigma \in(0,1)$ and $c, C>0$ then

$$
\liminf _{t \rightarrow \infty} \frac{1}{t} \mathrm{~N}_{\Omega, t A}(\lambda(t)) \geq \Phi_{\Omega}(|B|) .
$$

For strong fields this result guarantees the existence of approximately $\Phi_{\Omega}(|t B|)$ sub-exponentially small eigenvalues of the Pauli operator. The corresponding eigenfunctions, which we informally term approximate zero modes, can be viewed as a local version of the Aharonov-Casher zero modes. The latter are a dimension $\left\lfloor\left|\Phi_{\mathbb{R}^{2}}(t B)\right|\right\rfloor$ set of spin-definite zero energy bound states of the Pauli operator on $\mathbb{R}^{2}$; the spin is aligned with the dominate sign of $B$ (see [2] and [10]). In the strong field limit strong localisation should confine such states to regions where $B$ has its dominate sign; indeed such localisation of the Aharonov-Casher construction lies at the heart of our argument (see below and Section 4 for further details). A different manifestation of this localisation, relating to the ground state density of the Pauli operator on $\mathbb{R}^{2}$, was obtained in [7]. 
Remark 1.1. The magnetic potential $A$ (and hence the Pauli operator $\mathcal{P}_{\Omega, A}$ ) is not uniquely defined by $B$. If $\Omega$ is simply connected different choices of $A$ lead to unitarily equivalent Pauli operators (see Proposition 2.7) so the counting function $\mathrm{N}_{\Omega, A}$ will not depend on the particular choice of $A$. For more general regions this is no longer true; in this case our results hold independently of the choice of $A$.

Remark 1.2. It is possible to consider operators corresponding to non-Dirichlet boundary conditions. While there is a natural choice for a Neumann version of the magnetic Schrödinger operator (which has received particular attention in connection with the Ginzburg-Landau theory of superconductivity), it is less clear how one should define a Neumann version of the Pauli operator. One possibility would be to use the maximal closed extensions of $P_{A, \pm}$ in (1); however such an operator does not have a compact resolvent (even when $A \equiv 0$ ), leading to a very different class of spectral problems. Alternatively one could use (2) to define a "Neumann" Pauli operator in terms of the Neumann magnetic Schrödinger operator. With some additional restrictions on the regularity of $\Omega$ (such as having a Lipschitzian boundary) Theorems 1.1 and 1.2 can be extended to cover such operators (see Remark 2.6 for some further details). However the operator defined in this manner is not always non-negative (so cannot be the square of a Dirac operator). Only relatively crude estimates for the asymptotics of the size and number of negative eigenvalues follow from the immediate extensions to our results; in particular, for any $\varepsilon>0$ the number of eigenvalues below $-\varepsilon t$ is $o(t)$ as $t \rightarrow+\infty$. Further work would be needed to determine whether (the majority of) those eigenvalues guaranteed by Theorem 1.2 have small absolute value (so can be regarded as belonging to approximate zero modes).

Remark 1.3. When $B$ is constant the spectra of the Pauli and magnetic Schrödinger operators on $\mathbb{R}^{2}$ reduces to a set Landau levels. For non-constant fields this level structure is destroyed, with the typical exception of the zero energy level of the Pauli operator (the Aharonov-Casher zero modes). On the other hand the lower and upper bounds given by Theorem 1.1 will differ for any $\Lambda$ corresponding to a Landau level generated by a value at which $B$ is locally constant on some region (these are precisely the $\Lambda$ for which the set in (4) has non-zero measure). It is likely that a version of Theorem 1.2 could be extended to such cases. A related problem of eigenvalue accumulation near Landau levels after perturbation by a decaying electric potential has been considered; see [6] and references therein.

Precise definitions and various preliminary results are collected in Section 2; in particular, the Lichnerowicz formula (2) is justified (Proposition 2.4), a priori bounds on $\mathrm{N}_{\Omega, A}(\lambda)$ are obtained (Proposition 2.5) and gauge transformations are discussed (Proposition 2.7).

The proof of Theorem 1.1 is given in Section 3. This follows a standard localisation type argument (c.f., [3], [25]) using a sequence of piecewise constant approximations to $B$ based on increasingly fine tilings of $\Omega_{0}$ by squares (Section 3.2). The corresponding approximation results for quadratic forms are obtained in Section 3.3 while the necessary eigenvalue counting function results for constant fields on a square are given in Section 3.1 (these are taken almost directly from [3]). The bounds in Theorem 1.1 are finally pieced together in Section 3.4. 
Theorem 1.2 is justified in Section 4 by initially reducing the problem to the case of fields of constant sign on a disc (Section 4.1). Suitable test functions on the disc can be constructed from holomorphic functions with the help of the "real gauge" transformation introduced in [2]; these functions need to be cut-off at the boundary, a process which ultimately leads to a spectral problem on the circle (Section 4.2, with further technical details in Sections 4.3 and 4.4).

\section{Notation}

For a bounded open region $\Omega \subset \mathbb{R}^{2}$ we use $C(\Omega), C^{\alpha}(\Omega)$ and $\mathcal{O}(\Omega)$ to denote the space of continuous, Hölder continuous and holomorphic functions on $\Omega$, without restriction on behaviour near the boundary; we replace $\Omega$ with $\bar{\Omega}$ to indicate uniform versions of the same spaces. For $k \in \mathbb{N}_{0}$ we use $C^{k, \alpha}(\Omega)$ and $W^{k, 2}(\Omega)$ to denote the Hölder-Zygmund and Sobolev space consisting of functions with $k$ derivatives in $C^{\alpha}(\Omega)$ and $L^{2}(\Omega)$ respectively. The completion of $C_{0}^{\infty}(\Omega)$ in $W^{k, 2}(\Omega)$ is denoted by $W_{0}^{k, 2}(\Omega)$, while $C_{0}^{k}(\Omega)$ denotes the space of $k$-times continuously differentiable functions with compact support contained in $\Omega$. Unless otherwise indicated norms and inner-products are defined in the relevant $L^{2}$ sense.

The open disc with radius $R>0$ and centre $a \in \mathbb{R}^{2}$ is denoted $\mathbb{D}_{R}(a)$. When $a=0$ or $R=1$ these values are omitted; in particular, $\mathbb{D}$ is the open unit disc. We also set $(x)_{+}=\max \{x, 0\}$, the positive part of $x \in \mathbb{R}$.

General positive constants are denoted by $C$, with numerical subscripts used to keep track of particular constants in subsequent discussions.

\section{Preliminaries}

Let $A \in L_{\text {loc }}^{2}\left(\Omega, \mathbb{R}^{2}\right)$. Consider the Dirac operator, initially defined by

$$
\mathcal{D}_{A} u=\sigma \cdot P_{A} u=\left(\begin{array}{cc}
0 & P_{A,-} \\
P_{A,+} & 0
\end{array}\right)\left(\begin{array}{l}
u_{+} \\
u_{-}
\end{array}\right)
$$

for $u \in C_{0}^{\infty}\left(\Omega, \mathbb{C}^{2}\right)$. The operator $\mathcal{D}_{A}$ is densely defined and symmetric, hence closable; by a slight abuse of notation we will also denote the closure by $\mathcal{D}_{A}$.

Remark 2.1. Alternatively we can proceed by considering the operators $P_{A, \pm}$ separately. Initially densely defined on $C_{0}^{\infty}(\Omega)$ these operators satisfy $P_{A, \pm} \subseteq P_{A, \mp}^{*}$ and are hence closable. Using the same notation for the closures (6) then holds for all $u \in \operatorname{Dom}\left(\mathcal{D}_{A}\right)=\operatorname{Dom}\left(P_{A,+}\right) \times \operatorname{Dom}\left(P_{A,-}\right)$.

Define a quadratic form by

$$
\mathbf{p}_{\Omega, A}(u)=\left\|\mathcal{D}_{A} u\right\|^{2}=\left\|P_{A,+} u_{+}\right\|^{2}+\left\|P_{A,-} u_{-}\right\|^{2}, \quad u \in \operatorname{Dom}\left(\mathcal{D}_{A}\right) .
$$

Since $\mathcal{D}_{A}$ is a closed operator $\mathbf{p}_{\Omega, A}$ is a closed non-negative quadratic form. The Pauli operator on $\Omega$ with magnetic potential $A$ and Dirichlet boundary conditions is defined to be the corresponding self-adjoint operator given by the representation theorem; we'll use the notation $\mathcal{P}_{\Omega, A}$. 
Remark 2.2 (Case $A \equiv 0$ ). Since $\Omega$ is bounded $\left(\left\|\nabla_{1} u\right\|^{2}+\left\|\nabla_{2} u\right\|^{2}\right)^{1 / 2}$ gives an equivalent norm on the Sobolev space $W_{0}^{1,2}(\Omega)$ (see [1]). Also, for $u \in C_{0}^{\infty}(\Omega)$,

$\left\|P_{0, \pm} u\right\|^{2}=\left\|\nabla_{1} u\right\|^{2}+\left\|\nabla_{2} u\right\|^{2} \mp i \int_{\Omega}\left(\nabla_{1} \bar{u} \nabla_{2} u-\nabla_{2} \bar{u} \nabla_{1} u\right)=\left\|\nabla_{1} u\right\|^{2}+\left\|\nabla_{2} u\right\|^{2}$.

Completion then gives $\operatorname{Dom}\left(\mathcal{D}_{0}\right)=W_{0}^{1,2}\left(\Omega, \mathbb{C}^{2}\right)$ with $\mathbf{p}_{\Omega, 0}(u) \asymp\|u\|_{W_{0}^{1,2}\left(\Omega, \mathbb{C}^{2}\right)}^{2}$.

Remark 2.3. Since $P_{A, \pm} \bar{u}=-\overline{P_{-A, \mp} u}$ when $u \in C_{0}^{\infty}(\Omega)$ we get $\mathbf{p}_{\Omega, A}(\mathcal{J} u)=$ $\mathbf{p}_{\Omega,-A}(u)$ for all $u \in \operatorname{Dom}\left(\mathbf{p}_{\Omega, A}\right)=\operatorname{Dom}\left(\mathbf{p}_{\Omega,-A}\right)$, where $\mathcal{J}$ is the anti-linear isometric involution on $L^{2}\left(\Omega, \mathbb{C}^{2}\right)$ defined by

$$
\mathcal{J} u=\left(\begin{array}{l}
\overline{u_{-}} \\
\overline{u_{+}}
\end{array}\right), \quad u=\left(\begin{array}{l}
u_{+} \\
u_{-}
\end{array}\right) \in L^{2}\left(\Omega, \mathbb{C}^{2}\right)
$$

It follows that $\mathcal{J P}_{\Omega, A} \mathcal{J}=\mathcal{P}_{\Omega,-A}$, and so $\mathcal{P}_{\Omega, A}$ and $\mathcal{P}_{\Omega,-A}$ have the same spectrum.

In order to make use of results for Schrödinger operators we will need a rigorous form of the Lichnerowicz formula (2). We begin by introducing the magnetic Schrödinger operator in a way that parallels our introduction of the Pauli operator.

For $l=1,2$ we initially define the operator $P_{A, l}=-i \nabla_{l}-A_{l}$ on $C_{0}^{\infty}(\Omega)$. This operator is densely defined and symmetric, hence closable; by a slight abuse of notation we will also denote the closure by $P_{A, l}$. Setting

$$
\mathcal{H}_{A}(\Omega)=\operatorname{Dom}\left(P_{A, 1}\right) \cap \operatorname{Dom}\left(P_{A, 2}\right),
$$

the quadratic form defined by

$$
\mathbf{h}_{\Omega, A}(u)=\left\|P_{A, 1} u\right\|^{2}+\left\|P_{A, 2} u\right\|^{2}, \quad u \in \mathcal{H}_{A}(\Omega)
$$

is closed and non-negative. The magnetic Schrödinger operator on $\Omega$ with magnetic potential $A$ and Dirichlet boundary conditions is defined to be the corresponding self-adjoint operator given by the representation theorem; we'll use the notation $H_{\Omega, A}$.

Remark 2.4 (Case $A \equiv 0$ ). It is straightforward to see that $\mathcal{H}_{0}(\Omega)=W_{0}^{1,2}(\Omega)$ with $\mathbf{h}_{\Omega, 0}(u) \asymp\|u\|_{W_{0}^{1,2}(\Omega)}^{2}$ (c.f., Remark 2.2). Furthermore $H_{\Omega, 0}=-\Delta_{\Omega}$, the laplacian on $\Omega$ with Dirichlet boundary conditions.

We need to add a scalar potential to the operator $H_{\Omega, A}$. Since $H_{\Omega, A}$ is a semi-bounded self-adjoint operator this can be done conveniently via the standard KLMN construction if the scalar potential is relatively form bounded with respect to $H_{\Omega, A}$ with relative bound less than 1 (see [22], for example). If $V \in L_{\text {loc }}^{1}(\Omega)$ is a real-valued function then the form given by

$$
\mathbf{v}(u)=\langle u, V u\rangle
$$

is certainly defined for $u \in C_{0}^{\infty}(\Omega)$. To extend $\mathbf{v}$ to $\mathcal{H}_{0}(\Omega)=W_{0}^{1,2}(\Omega)$ we need to restrict $V$ to the Orlicz space $L \log L(\Omega)$. More precisely, introduce the $N$-function

$$
\mathscr{A}(t)=(t+1) \log (t+1)-t, \quad t \geq 0
$$


we then define $L \log L(\Omega)$ to be the Orlicz space $L_{\mathscr{A}}(\Omega)$ (see [1]). It is straightforward to check that $L^{p}(\Omega) \subset L_{\mathscr{A}}(\Omega) \subset L^{1}(\Omega)$ for any $p>1$. Now suppose $V \in L_{\mathscr{A}}(\Omega)$. By [26, Lemma 2.1] (see also Remark 2.5 below) $\mathbf{v}$ given by (7) then defines a bounded form on $W_{0}^{1,2}(\Omega)$, while the corresponding operator $T_{V}$ is compact. Viewing $T_{V}$ as multiplication by $V$ acting as a mapping $W_{0}^{1,2}(\Omega) \rightarrow\left(W_{0}^{1,2}(\Omega)\right)^{*}$, it follows that $V$ is relatively form compact and hence infinitesimally form bounded with respect to $H_{\Omega, 0}$. The KLMN construction can then be used to define $H_{\Omega, 0}-V$. Since the Dirichlet laplacian $-\Delta_{\Omega}=H_{\Omega, 0}$ has a compact resolvent (see [23], for example) the infinitesimal form boundedness of $V$ implies $H_{\Omega, 0}-V$ also has a compact resolvent (see [17]).

Remark 2.5. The results we need from [26] are mostly stated from the case of Neumann boundary conditions under the assumption that $\Omega$ has a Lipschitzian boundary. However it is easy to see that they also hold in the Dirichlet case for arbitrary bounded $\Omega$.

The results of the above discussion can be generalised to include a magnetic potential $A$ with the help of the diamagnetic inequality; a convenient form of the latter can be found in [15].

Proposition 2.1. Let $A \in L_{\mathrm{loc}}^{2}(\Omega)$ and $V \in L_{\mathscr{A}}(\Omega)$. Then (multiplication by) $V$ is an infinitesimally form bounded perturbation of $H_{\Omega, A}$. Furthermore the semibounded self-adjoint operator $H_{\Omega, A}-V$ (resulting from the KLMN construction) has a compact resolvent.

Proof. The discussion proceeding the result covers the case $A \equiv 0$. Using [15, Theorem 3.3; see also Remark 3.4(i)] it follows that $V$ is infinitesimally form bounded with respect to $H_{\Omega, A}$, while, for $t>0$,

$$
e^{-t\left(H_{\Omega, A}-V\right)} \preccurlyeq e^{-t\left(H_{\Omega, 0}-V\right)}
$$

(where $S \preccurlyeq T$ means that $S$ is dominated by $T$ ). However if $S \preccurlyeq T$ and $T$ is compact then $S$ must also be compact (see [5], [21]), while for a semi-bounded self-adjoint operator $Q, e^{-Q}$ is compact iff $Q$ has a compact resolvent. It follows that $H_{\Omega, A}-V$ has a compact resolvent.

Our a priori bounds for the counting function of the Pauli operator can be obtained from suitable bounds on the number of negative eigenvalues of $H_{\Omega, A}-V$. The latter will be obtained through a two step process; results from [26] allow us to estimate the counting function for $H_{\Omega, 0}-V$ under the condition that $V \in L_{\mathscr{A}}(\Omega)$, while the techniques of [24] allow us to use the diamagnetic inequality (see (8)) to generalise to $H_{\Omega, A}-V$.

Proposition 2.2. Let $A \in L_{\mathrm{loc}}^{2}(\Omega)$ and $V \in L_{\mathscr{A}}(\Omega)$. Then

$$
\#\left\{\lambda_{n}\left(H_{\Omega, A}-V\right) \leq 0\right\} \leq C_{1}\|V\|_{L_{\mathscr{A}}(\Omega)} .
$$

Proof. Since the positive and negative parts of any $V \in L_{\mathscr{A}}(\Omega)$ also belong to $L_{\mathscr{A}}(\Omega)$, while the addition of a positive scalar potential can only raise eigenvalues, it suffices to prove the result assuming $V \geq 0$. 
Now $\left(H_{\Omega, 0}\right)^{1 / 2}: \mathcal{H}_{0}(\Omega) \rightarrow L^{2}(\Omega)$ is an isomorphism (this is equivalent to the fact that $\mathbf{h}_{\Omega, 0}(u) \asymp\|u\|_{W_{0}^{1,2}(\Omega)}^{2}$ on $\mathcal{H}_{0}(\Omega)=W_{0}^{1,2}(\Omega)$; see Remark 2.4). Thus the expression $S_{V}=\left(\left(H_{\Omega, 0}\right)^{-1 / 2}\right)^{*} T_{V}\left(H_{\Omega, 0}\right)^{-1 / 2}$ defines a non-negative self-adjoint operator on $L^{2}(\Omega)$. By [26, Corollary 2.3] $T_{V}$ and hence $S_{V}$ belong to the weak first Schatten class with $\left\|S_{V}\right\|_{1, w} \leq C_{1,1}\|V\|_{L_{\mathscr{A}(\Omega)}}$ for some constant $C_{1,1}$; in other words

$$
0 \leq \lambda_{n}\left(S_{V}\right) \leq C_{1,1}\|V\|_{L_{\mathscr{A}(\Omega)}} n^{-1}, \quad n \in \mathbb{N} .
$$

A standard Birman-Schwinger type argument then gives

$$
\#\left\{\lambda_{n}\left(H_{\Omega, 0}-\gamma V\right) \leq 0\right\} \leq C_{1,1} \gamma\|V\|_{L_{\mathscr{A}}(\Omega)}, \quad \gamma \geq 0 .
$$

Denote the right hand side as $\mu(\gamma)$ and let $\widehat{\mu}$ be the Laplace transform of $\mu$; in particular $\gamma^{-1} \widehat{\mu}\left(\gamma^{-1}\right)=\mu(\gamma)$. Now (8) (with $V \equiv 0$ ) gives $e^{-t H_{\Omega, A}} \preccurlyeq e^{-t H_{\Omega, 0}}$. Using [24, Theorem 3] we then obtain

$$
\#\left\{\lambda_{n}\left(H_{\Omega, A}-\gamma V\right) \leq 0\right\} \leq e \gamma^{-1} \widehat{\mu}\left(\gamma^{-1}\right)=C_{1} \gamma\|V\|_{L_{\mathscr{A}}(\Omega)}, \quad \gamma \geq 0,
$$

where $C_{1}=e C_{1,1}$.

We can now compare the Pauli operator with the magnetic Schrödinger operator. We begin by looking at the corresponding forms. For any $A \in L_{\text {loc }}^{2}(\Omega)$ and $u \in C_{0}^{\infty}(\Omega)$ we can define $\mathbf{b}(u)$ to be the distribution $B=\nabla_{1} A_{2}-\nabla_{2} A_{1}$ acting on the test function $|u|^{2} \in C_{0}^{\infty}(\Omega)$. If $B \in L_{\mathrm{loc}}^{1}(\Omega)$ then

$$
\mathbf{b}(u)=\int_{\Omega} B|u|^{2}=\langle u, B u\rangle ;
$$

that is, $\mathbf{b}$ is just the form associated with the operator of multiplication by $B$.

Lemma 2.3. Let $A \in L_{\mathrm{loc}}^{2}(\Omega)$. If $u \in C_{0}^{\infty}\left(\Omega, \mathbb{C}^{2}\right)$ then

$$
\mathbf{p}_{\Omega, A}(u)=\mathbf{h}_{\Omega, A}\left(u_{+}\right)-\mathbf{b}\left(u_{+}\right)+\mathbf{h}_{\Omega, A}\left(u_{-}\right)+\mathbf{b}\left(u_{-}\right) .
$$

Proof. If $v \in C_{0}^{\infty}(\Omega)$ then $\left\|P_{A, \pm} v\right\|^{2}=\left\|P_{A, 1} v\right\|^{2}+\left\|P_{A, 2} v\right\|^{2} \mp 2 \operatorname{Im}\left\langle P_{A, 1} v, P_{A, 2} v\right\rangle$ while

$$
\begin{aligned}
& 2 \operatorname{Im}\left\langle P_{A, 1} v, P_{A, 2} v\right\rangle=\int_{\Omega}\left[-i \nabla_{1} \bar{v} \nabla_{2} v-\nabla_{1} \bar{v} A_{2} v+A_{1} \bar{v} \nabla_{2} v-i A_{1} A_{2}|v|^{2}\right. \\
& \left.+i \nabla_{2} \bar{v} \nabla_{1} v-A_{2} \bar{v} \nabla_{1} v+\nabla_{2} \bar{v} A_{1} v+i A_{2} A_{1}|v|^{2}\right] \\
& =\int_{\Omega}\left[-A_{2} \nabla_{1}|v|^{2}+A_{1} \nabla_{2}|v|^{2}\right] .
\end{aligned}
$$

The final expression is just the distribution $\nabla_{1} A_{2}-\nabla_{2} A_{1}=B$ acting on $|v|^{2}$. The result now follows from the definitions of $\mathbf{p}_{\Omega, A}, \mathbf{h}_{\Omega, A}$ and $\mathbf{b}$.

If $B \in L_{\mathscr{A}}(\Omega)$ the operators $H_{\Omega, A} \mp B$ can be defined as discussed above. The corresponding forms $\mathbf{h}_{\Omega, A} \mp \mathbf{b}$ have core $C_{0}^{\infty}(\Omega)$, while $C_{0}^{\infty}\left(\Omega, \mathbb{C}^{2}\right)$ is a core for $\mathbf{p}_{\Omega, A}$. The previous result then gives $\operatorname{Dom}\left(\mathbf{p}_{\Omega, A}\right)=\mathcal{H}_{A}\left(\Omega, \mathbb{C}^{2}\right)$, with (9) extending to all $u \in \mathcal{H}_{A}\left(\Omega, \mathbb{C}^{2}\right)$. The operator identity (2) now follows, allowing Proposition 2.1 to be applied to $\mathcal{P}_{\Omega, A}$; we summarise what we need as follows. 
Proposition 2.4. Suppose $A \in L_{\text {loc }}^{2}(\Omega)$ with $B \in L_{\mathscr{A}}(\Omega)$. Then the Lichnerowicz formula (2) holds as an operator identity for the Pauli and magnetic Schrödinger operators with Dirichlet boundary conditions. Furthermore $\mathcal{P}_{\Omega, A}$ has a compact resolvent and hence discrete spectrum.

Proposition 2.2 can now be used to obtain a priori bounds on $\mathrm{N}_{\Omega, A}(\lambda)$. However we will need uniform versions of these bounds for sub-regions of $\Omega$. If $\Omega^{\prime} \subseteq$ $\Omega$ is open we can restrict $A$ to $\Omega^{\prime}$ and consider the Pauli operator $\mathcal{P}_{\Omega^{\prime}, A}$ with corresponding counting function $\mathrm{N}_{\Omega^{\prime}, A}(\lambda)$. Using $\chi_{\Omega^{\prime}}$ to denote the characteristic function for $\Omega^{\prime}$ Proposition 2.4 and a simple variational argument then give

$$
\mathrm{N}_{\Omega^{\prime}, A}(\lambda) \leq \#\left\{\lambda_{n}\left(H_{\Omega, A}-(\lambda+B) \chi_{\Omega^{\prime}}\right) \leq 0\right\}+\#\left\{\lambda_{n}\left(H_{\Omega, A}-(\lambda-B) \chi_{\Omega^{\prime}}\right) \leq 0\right\},
$$

for any $\lambda \in \mathbb{R}$. However $\left\|(\lambda \pm B) \chi_{\Omega^{\prime}}\right\|_{L_{\mathscr{A}}(\Omega)} \leq\|B\|_{L_{\mathscr{A}}\left(\Omega^{\prime}\right)}+|\lambda|\|1\|_{L_{\mathscr{A}}\left(\Omega^{\prime}\right)}$, so Proposition 2.2 now completes the following.

Proposition 2.5. Suppose $A \in L_{\mathrm{loc}}^{2}(\Omega)$ with $B \in L_{\mathscr{A}}(\Omega)$. Then

$$
\mathrm{N}_{\Omega^{\prime}, A}(\lambda) \leq 2 C_{1}\left(\|B\|_{L_{\mathscr{A}}\left(\Omega^{\prime}\right)}+|\lambda|\|1\|_{L_{\mathscr{A}}\left(\Omega^{\prime}\right)}\right)
$$

for any open $\Omega^{\prime} \subseteq \Omega$; the constant $C_{1}$ may depend on $\Omega$ but not on $\Omega^{\prime}$.

The magnetic potentials $A, A^{\prime} \in L_{\text {loc }}^{2}(\Omega)$ are gauge equivalent if $A^{\prime}=A+\nabla \psi$ for some $\psi \in W_{\text {loc }}^{1,2}(\Omega)$. It follows that $\operatorname{curl} A^{\prime}=\operatorname{curl} A$ (as distributions), so $A^{\prime}$ and $A$ generate the same magnetic field. The converse is not generally true; a topological condition on $\Omega$ is also required. The following is a particular case of [19, Lemma 1.1].

Lemma 2.6. Suppose $\Omega$ is simply connected. If $A, A^{\prime} \in L_{\mathrm{loc}}^{2}(\Omega)$ satisfy $\operatorname{curl} A^{\prime}=$ $\operatorname{curl} A$ (as distributions) then there exists $\psi \in W_{\mathrm{loc}}^{1,2}(\Omega)$ with $A^{\prime}=A+\nabla \psi$.

Now suppose $A, A^{\prime} \in L_{\text {loc }}^{2}(\Omega)$ are gauge equivalent and $V \in L_{\mathscr{A}}(\Omega)$ (so that the operators $H_{\Omega, A}-V$ and $H_{\Omega, A^{\prime}}-V$ correspond to the closures of the semibounded forms $\mathbf{h}_{\Omega, A}-\mathbf{v}$ and $\mathbf{h}_{\Omega, A^{\prime}}-\mathbf{v}$ on $\left.C_{0}^{\infty}(\Omega)\right)$. Choosing $\psi \in W_{\text {loc }}^{1,2}(\Omega)$ with $A^{\prime}=A+\nabla \psi$, the argument given for the proof of [19, Theorem 1.2] then shows the unitary operator $U_{\psi}$ of multiplication by $e^{i \psi}$ gives a unitary equivalence $H_{\Omega, A^{\prime}}-V=U_{\psi}\left(H_{\Omega, A}-V\right) U_{\psi}^{*}$ (note that [19, Theorem 1.2] is stated for $\Omega=\mathbb{R}^{2}$ and only assumes $\operatorname{curl} A=\operatorname{curl} A^{\prime}$; however the former is only used to guarantee the existence of $\psi$, after which the proof easily adapts to cover arbitrary $\Omega$ ). Coupled with Proposition 2.4 and Lemma 2.6 we arrive at the following.

Proposition 2.7. Suppose $A, A^{\prime} \in L_{\mathrm{loc}}^{2}(\Omega)$ satisfy $A^{\prime}=A+\nabla \psi$ for some $\psi \in$ $W_{\mathrm{loc}}^{1,2}(\Omega)$ (which follows from the condition $\operatorname{curl} A^{\prime}=\operatorname{curl} A$ when $\Omega$ is simply connected). Also suppose $B \in L_{\mathscr{A}}(\Omega)$. Then $U_{\psi}$ gives the unitary equivalence $\mathcal{P}_{\Omega, A^{\prime}}=U_{\psi} \mathcal{P}_{\Omega, A} U_{\psi}^{*} ;$ in particular, $\mathcal{P}_{\Omega, A}$ and $\mathcal{P}_{\Omega, A^{\prime}}$ have the same spectrum. 
Remark 2.6 (Maximal operators). For $l=1,2$ the operator $P_{A, l}$ is the minimal closed extension of the magnetic momentum operator initially defined on $C_{0}^{\infty}(\Omega)$. The corresponding maximal closed extension thus satisfies $P_{A, l}^{\max }=P_{A, l}^{*}$. The closed non-negative quadratic form $\mathbf{h}_{\Omega, A}^{\max }(u)=\left\|P_{A, 1}^{\max } u\right\|^{2}+\left\|P_{A, 2}^{\max } u\right\|^{2}$ can be used to define the magnetic Schrödinger operator on $\Omega$ with magnetic potential $A$ and Neumann boundary conditions (see [15] for further discussion of this operator).

Neumann versions of Propositions 2.1 and 2.2 are possible if we assume $\Omega$ has some additional regularity. In all cases it is sufficient to assume the existence of a linear extension operator which is continuous as a map $W^{k, 2}(\Omega) \rightarrow W^{k, 2}\left(\mathbb{R}^{2}\right)$ for $k=0,1$ (such operators exist if $\Omega$ has a Lipschitzian boundary). Assuming $\Omega$ satisfies such a condition and $B \in L_{\mathscr{A}}(\Omega)$ we can use $(2)$ to define a "Neumann" Pauli operator from the Neumann magnetic Schrödinger operator; denote this operator by $\mathcal{P}_{\Omega, A}^{\prime}$, with corresponding form $\mathbf{p}_{\Omega, A}^{\prime}$. It is then possible to extend Theorems 1.1 and 1.2 to cover $\mathcal{P}_{\Omega, A}^{\prime}$, for the most part simply by using a combination of variational arguments (note that $\mathbf{p}_{\Omega, A}^{\prime}$ is an extension of $\mathbf{p}_{\Omega, A}$ ) and straightforward modifications to the given proofs; the most notable exception is Proposition 2.5 and its application where, to retain uniformity in $\Omega^{\prime}$, one is forced to consider operators with mixed boundary conditions (Neumann on $\partial \Omega^{\prime} \cap \partial \Omega$ and Dirichlet on $\left.\partial \Omega^{\prime} \backslash \partial \Omega\right)$.

We complete this section by considering some basic properties of the auxiliary functions $\nu^{ \pm}$. If $b \neq 0$ then (3) gives $\nu(b, \lambda)=0$ when $\lambda<0$, while

$$
\nu(b, \lambda)=(2 m+1) \frac{|b|}{2 \pi} \leq \frac{1}{2 \pi}(\lambda+|b|)
$$

when $2 m|b|<\lambda<2(m+1)|b|$ for some $m \in \mathbb{N}_{0}$. It follows that $\nu$ is locally bounded, so $\nu^{ \pm}$are well defined locally bounded functions on $\mathbb{R}^{2}$. Furthermore

$$
0 \leq \nu^{ \pm}(b, \lambda) \leq \frac{1}{2 \pi}(|b|+|\lambda|), \quad b, \lambda \in \mathbb{R} .
$$

In particular (10) ensures the integrals appearing in Theorem 1.1 are finite whenever $B \in L^{1}(\Omega)$. We further note that $\nu^{ \pm}$are homogeneous of degree 1 while, for any $b, \lambda \in \mathbb{R}$, we have $\nu^{ \pm}(b, \lambda)=0$ if $\lambda<0, \nu^{ \pm}(0, \lambda)=\lambda /(2 \pi)$ if $\lambda \geq 0\left(\nu^{ \pm}\right.$ are actually continuous at $b=0), \nu^{-}(b, 0)=0$ and $\nu^{+}(b, 0)=|b| /(2 \pi)$. The final identity reduces the upper bound in Theorem 1.1 to (5) when $\Lambda=0$.

\section{General Asymptotics}

\subsection{Constant field on a square}

For $R>0$ and $b \in \mathbb{R}$ let $\mathcal{P}_{R, b}$ denote a Pauli operator on the square $(0, R)^{2}$ with Dirichlet boundary conditions and corresponding to a constant magnetic field $b$. One choice for the magnetic potential is $A(x)=b\left(-x_{2}, x_{1}\right) / 2$, while $(0, R)^{2}$ is simply connected so Proposition 2.7 shows that any other choice leads to a unitarily equivalent operator. Thus the eigenvalue counting function

$$
\mathrm{N}_{R, b}(\lambda)=\#\left\{\lambda_{n}\left(\mathcal{P}_{R, b}\right) \leq \lambda\right\}
$$


(counting with multiplicity) depends only on $R, b$ and $\lambda$. We can estimate $\mathrm{N}_{R, b}(\lambda)$ using the auxiliary function introduced in (3).

Proposition 3.1. For any $\lambda, b \in \mathbb{R}$ and $\rho \in(0,1)$ we have

$$
R^{2}(1-\rho)^{2} \nu^{+}\left(b, \lambda-C_{2} R^{-2} \rho^{-2}\right) \leq \mathrm{N}_{R, b}(\lambda) \leq R^{2} \nu^{+}(b, \lambda),
$$

where $C_{2}$ can be chosen as an absolute constant.

Proof. Let $H_{R,|b|}$ denote a Dirichlet magnetic Schrödinger operator on $(0, R)^{2}$ corresponding to the constant field $|b|$, and let $\mathrm{n}_{R,|b|}(\lambda)=\#\left\{\lambda_{n}\left(H_{R,|b|}\right) \leq \lambda\right\}$ denote the associated eigenvalue counting function (including multiplicity). Using Remark 2.3 and Proposition 2.4 we then get

$$
\mathrm{N}_{R, b}(\lambda)=\mathrm{N}_{R,|b|}(\lambda)=\mathrm{n}_{R,|b|}(\lambda+|b|)+\mathrm{n}_{R,|b|}(\lambda-|b|) .
$$

On the other hand, [3, Theorem 3.1] gives an absolute constant $C_{2}$ such that

$$
R^{2}(1-\rho)^{2} \mu\left(|b|, \lambda-C_{2} R^{-2} \rho^{-2}\right) \leq \mathrm{n}_{R,|b|}(\lambda) \leq R^{2} \mu(|b|, \lambda),
$$

where $\mu(|b|, \lambda)=0$ for $\lambda<0, \mu(0, \lambda)=\lambda /(4 \pi)$ for $\lambda \geq 0$, and

$$
\mu(|b|, \lambda)=\frac{|b|}{2 \pi} \#\left\{m \in \mathbb{N}_{0}:(2 m+1)|b| \leq \lambda\right\}
$$

when $b \neq 0$. Since $\mu(|b|, \lambda+|b|)+\mu(|b|, \lambda-|b|)=\nu^{+}(b, \lambda)$ the result follows.

\subsection{Localisation}

We want to approximate the field $B \in L_{\mathscr{A}}(\Omega) \cap C\left(\Omega_{0}\right)$ by a sequence of fields which take constant values on squares within $\Omega_{0}$. This approximation can only be made sufficiently good where $B$ is continuous (continuity is used when making the corresponding approximation to the potential; see Lemma 3.3). In turn this necessitates a degree of delicacy in the choice of the squares and the rate at which they approach the boundary of $\Omega_{0}$ (see Lemma 3.2).

For each $\delta>0$ set

$$
\Omega_{\delta}=\left\{x \in \Omega_{0}: \overline{\mathbb{D}}_{\delta}(x) \subset \Omega_{0}\right\} .
$$

Clearly $\Omega_{\delta}$ is open, $\Omega_{\delta} \subset \subset \Omega_{\delta^{\prime}}$ whenever $0 \leq \delta^{\prime}<\delta$ (recall that $\Omega_{0}$ is bounded) and

$$
\Omega_{0}=\bigcup_{\delta>0} \Omega_{\delta} .
$$

Since $B \in C\left(\Omega_{0}\right)$ it follows that $B \in C\left(\overline{\Omega_{\delta}}\right)$ for any $\delta>0$.

Lemma 3.2. We can find a strictly increasing sequence $\left(k_{l}\right)_{l \in \mathbb{N}_{0}}$ in $\mathbb{N}$ and, for each $k \geq k_{0}$, a finite indexing set $J_{k}$ and collection of disjoint open squares $S^{k, j}$, $j \in J_{k}$, of side length $2^{-k}$ with the following properties: setting

$$
\Omega^{k}=\operatorname{int} \overline{\bigcup_{j \in J_{k}} S^{k, j}}
$$

then, for each $l \in \mathbb{N}_{0}$ and $k_{l} \leq k<k_{l+1}$, 
(i) $\Omega_{2^{-l+1}} \subseteq \Omega^{k} \subset \subset \Omega_{2^{-l}}$.

(ii) For any $x, y \in \Omega^{k}$ with $|x-y| \leq 2^{-k-1 / 2}$ we have $|B(x)-B(y)| \leq 2^{-l}$.

Each of the squares $S^{k, j}$ will be a translate of $\left(0,2^{-k}\right)^{2}$. We will use $\widehat{S}^{k, j}$ and $\bar{S}^{k, j}$ to denote the corresponding translates of $\left[0,2^{-k}\right)^{2}$ and $\left[0,2^{-k}\right]^{2}$; thus $\bar{S}^{k, j}$ is just the closure of $S^{k, j}$ while $S^{k, j} \subset \widehat{S}^{k, j} \subset \bar{S}^{k, j}$. The set $\Omega^{k}$ is essentially the union of the squares $S^{k, j}, j \in J_{k}$, together with any edges lying between two squares. More precisely

$$
\Omega^{k}=\operatorname{int} \bigcup_{j \in J_{k}} \bar{S}^{k, j}=\operatorname{int} \bigcup_{j \in J_{k}} \widehat{S}^{k, j}
$$

in particular, each $x \in \Omega^{k}$ belongs to $\widehat{S}^{k, j}$ for a unique $j \in J_{k}$.

Proof of Lemma 3.2. For each $k \in \mathbb{N}_{0}$ and $\delta>0$ set

$$
d_{k}(\delta)=\sup \left\{|B(x)-B(y)|: x, y \in \Omega_{\delta},|x-y| \leq 2^{-k-1 / 2}\right\} .
$$

Since $B \in C\left(\overline{\Omega_{\delta}}\right)$ we have $d_{k}(\delta) \rightarrow 0$ as $k \rightarrow \infty$ (for fixed $\delta$ ). Hence we can find a strictly increasing sequence $\left(k_{l}\right)_{l \in \mathbb{N}_{0}}$ in $\mathbb{N}$ with $d_{k_{l}}\left(2^{-l}\right) \leq 2^{-l}$ and $k_{l}>l$ for each $l \in \mathbb{N}_{0}$.

Let $k \geq k_{0}$ and choose $l \in \mathbb{N}_{0}$ so that $k_{l} \leq k<k_{l+1}$. Consider the tiling of $\mathbb{R}^{2}$ by copies of the square $\left[0,2^{-k}\right)^{2}$ which have been translated so that the corners lie on points of the lattice $\left(2^{-k} \mathbb{Z}\right)^{2}$. Let $\widehat{S}^{k, j}$ for $j \in J_{k}$ denote the collection of squares from this tiling whose closure lies entirely within $\Omega_{2^{-l}}$. Set $S^{k, j}=\operatorname{int}\left(\widehat{S}^{k, j}\right)$ for $j \in J_{k}$ and define $\Omega^{k}$ by (12). Clearly $\Omega^{k} \subset \subset \Omega_{2^{-l}}$. Now suppose $x \in \Omega_{2^{-l+1}}$, so $\overline{\mathbb{D}}_{2^{-l+1}}(x) \subset \Omega_{0}$. Let $\bar{S}$ be the closure of any square from the tiling with $x \in \bar{S}$ and let $y \in \bar{S}$. Then $|x-y| \leq 2^{-k+1 / 2}<2^{-l}$ (since $k \geq k_{l}>l$ ) so $\overline{\mathbb{D}}_{2^{-l}}(y) \subset$ $\overline{\mathbb{D}}_{2^{-l+1}}(x) \subset \Omega_{0}$ and hence $y \in \Omega_{2^{-l}}$. Thus $\bar{S} \subset \Omega_{2^{-l}}$ and so $S \in\left\{S^{k, j}: j \in J_{k}\right\}$. It follows that $x \in \Omega^{k}$. Finally, if $x, y \in \Omega^{k}$ with $|x-y| \leq 2^{-k-1 / 2}$ then $x, y \in \Omega_{2^{-l}}$ with $|x-y| \leq 2^{-k_{l}-1 / 2}\left(\right.$ since $\left.k \geq k_{l}\right)$, so

$$
|B(x)-B(y)| \leq d_{k_{l}}\left(2^{-l}\right) \leq 2^{-l}
$$

(recall the defining properties of $k_{l}$ ).

By Lemma 3.2(i) and (11) we get $\bigcap_{k>k_{0}}\left(\Omega \backslash \overline{\Omega^{k}}\right)=\bigcap_{\delta>0}\left(\Omega \backslash \Omega_{\delta}\right)=\Omega \backslash \Omega_{0}$. Since $|\Omega|<\infty$ and $\left|\Omega \backslash \Omega_{0}\right|=0$ it follows that

$$
\left|\Omega \backslash \overline{\Omega^{k}}\right| \rightarrow 0 \text { as } k \rightarrow \infty .
$$

For $k \geq k_{0}$ set $\beta_{k}=2^{-l}$ where $l \in \mathbb{N}_{0}$ is maximal such that $k_{l} \leq k$. Lemma 3.2 implies $\left(\beta_{k}\right)_{k \geq k_{0}}$ is a non-increasing positive sequence with $\beta_{k} \rightarrow 0$ as $k \rightarrow \infty$ while, for each $k \geq k_{0}$,

$$
|B(x)-B(y)| \leq \beta_{k} \text { whenever } x, y \in \Omega^{k} \text { with }|x-y| \leq 2^{-k-1 / 2} .
$$

For $k \geq k_{0}$ and $j \in J_{k}$ set $b^{k, j}=B(x)$ where $x$ is the centre of the square $S^{k, j}$. 
Lemma 3.3. For any $k \geq k_{0}$ and $j \in J_{k}$ we can find a potential $A^{k, j} \in L_{\mathrm{loc}}^{2}\left(S^{k, j}\right)$ with $\operatorname{curl} A^{k, j}=b^{k, j}$ and $\left\|A-A^{k, j}\right\|_{L^{\infty}\left(S^{k, j}\right)} \leq \alpha_{k}$ where $\alpha_{k}=2^{-k-3 / 2} \beta_{k}$.

In particular, the potential $A^{k, j}$ generates the constant field $b^{k, j}$ on $S^{k, j}$.

Proof. Let $k \geq k_{0}$ and $j \in J_{k}$. For convenience centre $S^{k, j}$ at the origin and set

$$
\widetilde{A}_{1}^{k, j}(x)=-\frac{1}{2} \int_{0}^{x_{2}} B\left(x_{1}, t\right) d t \quad \text { and } \quad \widetilde{A}_{2}^{k, j}(x)=\frac{1}{2} \int_{0}^{x_{1}} B\left(t, x_{2}\right) d t .
$$

Then $\widetilde{A}^{k, j}, \nabla_{2} \widetilde{A}_{1}^{k, j}$ and $\nabla_{1} \widetilde{A}_{2}^{k, j}$ are all continuous on $S^{k, j}$ (since $B$ is continuous) while curl $\widetilde{A}^{k, j}=B=\operatorname{curl} A$. By Lemma 2.6 we can then find $\psi^{k, j} \in W_{\mathrm{loc}}^{1,2}\left(S^{k, j}\right)$ with $A-\widetilde{A}^{k, j}=\nabla \psi^{k, j}$ on $S^{k, j}$. Now set

$$
A_{1}^{k, j}(x)=\nabla_{1} \psi^{k, j}-\frac{1}{2} b^{k, j} x_{2} \quad \text { and } \quad A_{2}^{k, j}(x)=\nabla_{2} \psi^{k, j}+\frac{1}{2} b^{k, j} x_{1} .
$$

Then $A^{k, j} \in L_{\text {loc }}^{2}\left(S^{k, j}\right)$ with curl $A^{k, j}=b^{k, j}=B(0)$. For $x \in S^{k, j}$ (14) leads to

$$
\left|A_{1}(x)-A_{1}^{k, j}(x)\right|=\left|\frac{1}{2} \int_{0}^{x_{2}}\left(B\left(x_{1}, t\right)-B(0)\right) d t\right| \leq 2^{-k-2} \beta_{k}
$$

(note that $\left|x_{2}\right| \leq 2^{-k-1}$ ). Clearly a similar estimate holds for $A_{2}-A_{2}^{k, j}$.

Also let $\chi_{j}$ denote the characteristic function of the set $\widehat{S}^{k, j} \cap \Omega^{k}$, restricted to $\Omega$. Define a piecewise constant field $B^{k}: \Omega \rightarrow \mathbb{R}$ by

$$
B^{k}=\sum_{j \in J_{k}} b^{k, j} \chi_{j}
$$

The approximation $B^{k}$ converges to $B$ pointwise on $\Omega_{0}$ as $k \rightarrow \infty$; it will be helpful to combine this convergence with the Fatou-Lebesgue theorem as follows.

Lemma 3.4. Suppose $k(t) \in \mathbb{N}$ and $\Gamma(t) \in \mathbb{R}$ for each $t>0$ with $k(t) \rightarrow \infty$ and $\Gamma(t) \rightarrow \Gamma$ as $t \rightarrow \infty$. Then

$$
\liminf _{t \rightarrow \infty} \int_{\Omega} \nu^{+}\left(B^{k(t)}, \Gamma(t)\right) \geq \int_{\Omega} \nu^{-}(B, \Gamma)
$$

and

$$
\limsup _{t \rightarrow \infty} \int_{\Omega} \nu^{+}\left(B^{k(t)}, \Gamma(t)\right) \leq \int_{\Omega} \nu^{+}(B, \Gamma) .
$$

Proof. Let $k \geq k_{0}$. If $x \in \Omega^{k}$ then $x \in \widehat{S}^{k, j}$ for some $j \in J_{k}$ and so $B^{k}(x)=b^{k, j}=$ $B\left(x_{0}\right)$ where $x_{0}$ denotes the centre of $S^{k, j}$. However $\left|x-x_{0}\right| \leq 2^{-k-1 / 2}$ so

$$
\left|B^{k}(x)-B(x)\right|=\left|B\left(x_{0}\right)-B(x)\right| \leq \beta_{k}
$$

by (14). Since $\beta_{k} \leq 1$ it follows that $\left|B^{k}(x)\right| \leq|B(x)|+1$. This estimate is also valid when $x \notin \Omega^{k}$ since $B^{k}(x)=0$ in this case. For any $\lambda \in \mathbb{R}$ (10) now gives

$$
0 \leq \nu^{+}\left(B^{k}, \lambda\right) \leq \frac{1}{2 \pi}(|B|+1+|\lambda|) .
$$


If $x \in \Omega_{0}$ then (11) and Lemma 3.2(i) imply $x \in \Omega^{k}$ for all sufficiently large $k$, so $B^{k}(x) \rightarrow B(x)$ as $k \rightarrow \infty$ by (16). Since $\left|\Omega \backslash \Omega_{0}\right|=0$ it follows that $B^{k}$ converges to $B$ pointwise almost everywhere on $\Omega$ as $k \rightarrow \infty$. The result now follows from the Fatou-Lebesgue theorem (recall that $\nu^{-} \leq \nu^{+}$while $\nu^{-}$and $\nu^{+}$ are lower and upper semi-continuous respectively).

\subsection{Quadratic form estimates}

Recall the notation introduced in Lemma 3.2. For each $k \geq k_{0}$ and $\delta>0$ set

$$
\widetilde{R}_{\delta}^{k}=\bigcap_{j \in J_{k}} \bigcup_{x \in \mathbb{R}^{2} \backslash S^{k, j}} \mathbb{D}_{2^{-k} \delta}(x) \quad \text { and } \quad R_{\delta}^{k}=\widetilde{R}_{\delta}^{k} \cap \Omega .
$$

Thus $R_{\delta}^{k}$ is an open subset of $\Omega$ which contains all of $\Omega \backslash \Omega^{k}$, together with a $2^{-k} \delta$-neighbourhood of the boundary of each square $S^{k, j}, j \in J_{k}$. In particular, any point of $R_{\delta}^{k} \cap \overline{\Omega^{k}}$ must lie in $\bar{S}^{k, j}$ for some $j \in J_{k}$, at a distance of less than $2^{-k} \delta$ from the boundary (of $S^{k, j}$ ). Since $S^{k, j}$ has side length $2^{-k}$ it follows that $\left|R_{\delta}^{k} \cap \overline{\Omega^{k}}\right| \leq\left|J_{k}\right| 2^{-2 k+2} \delta$. However $\left|J_{k}\right| \leq 2^{2 k}|\Omega|$ (since the disjoint squares $S^{k, j}$, $j \in J_{k}$ are all contained in $\Omega$ ) so

$$
\left|R_{\delta}^{k}\right| \leq\left|\Omega \backslash \overline{\Omega^{k}}\right|+\left|R_{\delta}^{k} \cap \overline{\Omega^{k}}\right| \leq\left|\Omega \backslash \overline{\Omega^{k}}\right|+4|\Omega| \delta .
$$

We will need a partition of unity which is subordinate to the cover of $\mathbb{R}^{2}$ given by $\widetilde{R}_{\delta}^{k}$ and $S^{k, j}$ for $j \in J_{k}$. Using a standard construction we can find $\phi \in C^{\infty}\left(\mathbb{R}^{2}\right)$ and $\psi_{j} \in C_{0}^{\infty}\left(S^{k, j}\right)$ for $j \in J_{k}$ so that

$$
\phi^{2}+\sum_{j \in J_{k}} \psi_{j}^{2}=1 \quad \text { and } \quad|\nabla \phi|^{2}+\sum_{j \in J_{k}}\left|\nabla \psi_{j}\right|^{2} \leq C_{3} 2^{2 k} \delta^{-2},
$$

where the constant $C_{3}$ can be chosen independently of $k$ and $\delta$ (note that, $\nabla \psi_{j}$ is non-zero only in a $2^{-k} \delta$-neighbourhood of the boundary of $S^{k, j}$ ). Also recall the approximating magnetic potential $A^{k, j}$ introduced in Lemma 3.3 .

Proposition 3.5. Let $t>0$ and $\varepsilon \in(0,1)$. Then

$$
\mathbf{p}_{\Omega, t A}(u) \leq(1-\varepsilon)^{-1} \sum_{j \in J_{k}} \mathbf{p}_{S^{k, j}, t A^{k, j}}\left(u_{j}\right)+\varepsilon^{-1} t^{2} \alpha_{k}^{2}\|u\|^{2}
$$

whenever $u=\sum_{j \in J_{k}} u_{j}$ with $u_{j} \in C_{0}^{\infty}\left(S^{k, j}, \mathbb{C}^{2}\right), j \in J_{k}$. On the other hand,

$\mathbf{p}_{\Omega, t A}(u) \geq \mathbf{p}_{R_{\delta}^{k}, t A}(\phi u)+(1+\varepsilon)^{-1} \sum_{j \in J_{k}} \mathbf{p}_{S^{k, j}, t A^{k, j}}\left(\psi_{j} u\right)-\left(\varepsilon^{-1} t^{2} \alpha_{k}^{2}+C_{3} 2^{2 k} \delta^{-2}\right)\|u\|^{2}$

for any $u \in \mathcal{H}_{t A}\left(\Omega, \mathbb{C}^{2}\right)$.

Proof. Firstly let $j \in J_{k}$ and suppose $w \in C_{0}^{\infty}\left(S^{k, j}, \mathbb{C}^{2}\right)$. Then

$$
\mathbf{p}_{S^{k, j}, t A}(w)=\left\|\mathcal{D}_{t A} w\right\|^{2}=\left\|\mathcal{D}_{t A^{k, j}} w-t \sigma \cdot\left(A-A^{k, j}\right) w\right\|^{2} .
$$


Now $\left|\sigma .\left(A-A^{k, j}\right) \xi\right|=\left|A-A^{k, j}\right||\xi|$ for any $\xi \in \mathbb{C}^{2}$, so Lemma 3.3 gives

$$
\left\|\sigma \cdot\left(A-A^{k, j}\right) w\right\|^{2} \leq\left\|A-A^{k, j}\right\|_{L^{\infty}\left(S^{k, j}\right)}^{2}\|w\|^{2} \leq \alpha_{k}^{2}\|w\|^{2} .
$$

Basic norm estimates then lead to

$$
\begin{aligned}
(1+\varepsilon)^{-1} & \mathbf{p}_{S^{k, j}, t A^{k, j}}(w)-\varepsilon^{-1} t^{2} \alpha_{k}^{2}\|w\|^{2} \\
& \leq \mathbf{p}_{S^{k, j}, t A}(w) \leq(1-\varepsilon)^{-1} \mathbf{p}_{S^{k, j}, t A^{k, j}}(w)+\varepsilon^{-1} t^{2} \alpha_{k}^{2}\|w\|^{2}
\end{aligned}
$$

Taking completions extends this estimate to any $w \in \mathcal{H}_{t A^{k, j}}\left(S^{k, j}, \mathbb{C}^{2}\right)$.

If $u=\sum_{j \in J_{k}} u_{j}$ with $u_{j} \in C_{0}^{\infty}\left(S^{k, j}, \mathbb{C}^{2}\right), j \in J_{k}$, then

$$
\|u\|^{2}=\sum_{j \in J_{k}}\left\|u_{j}\right\|^{2} \quad \text { and } \quad \mathbf{p}_{\Omega, t A}(u)=\sum_{j \in J_{k}} \mathbf{p}_{S^{k, j}, t A}\left(u_{j}\right)
$$

since the $S^{k, j}$ 's are disjoint; (19) now follows from the second estimate in (21).

Now suppose $v \in C_{0}^{\infty}(\Omega)$. Enlarge $J_{k}$ to $J_{k}^{\prime}$ to include an index for $\phi$ and let $j \in J_{k}^{\prime}$. Since $P_{t A}\left(\psi_{j} v\right)=\psi_{j} P_{t A} v-i\left(\nabla \psi_{j}\right) v$ the first part of (18) gives

$$
\sum_{j \in J_{k}^{\prime}}\left|P_{t A, l}\left(\psi_{j} v\right)\right|^{2}=\left|P_{t A, l} v\right|^{2}+\sum_{j \in J_{k}^{\prime}}\left(\nabla_{l} \psi_{j}\right)^{2}|v|^{2}
$$

for $l=1,2$. Integration over $\Omega$ and the second part of (18) then lead to

$$
\mathbf{h}_{\Omega, t A}(v) \geq \sum_{j \in J_{k}^{\prime}} \mathbf{h}_{\Omega, t A}\left(\psi_{j} v\right)-C_{3} 2^{2 k} \delta^{-2}\|v\|^{2} .
$$

An easy calculation also gives $\mathbf{b}(v)=\sum_{j \in J_{k}^{\prime}} \mathbf{b}\left(\psi_{j} v\right)$. Hence

$$
\mathbf{p}_{\Omega, t A}(u) \geq \mathbf{p}_{R_{\delta}^{k}, t A}(\phi u)+\sum_{j \in J_{k}} \mathbf{p}_{S^{k, j}, t A}\left(\psi_{j} u\right)-C_{3} 2^{2 k} \delta^{-2}\|u\|^{2}
$$

for any $u \in C_{0}^{\infty}\left(\Omega, \mathbb{C}^{2}\right)$. For such $u(20)$ now follows from the first estimate in (21) and the fact that $\sum_{j \in J_{k}}\left\|\psi_{j} u\right\|^{2}=\|u\|^{2}-\|\phi u\|^{2} \leq\|u\|^{2}$. Taking completions then gives (20) for all $u \in \mathcal{H}_{t A}\left(\Omega, \mathbb{C}^{2}\right)$.

Standard variational arguments allow us to use the quadratic form estimates of Proposition 3.5 to obtain corresponding bounds on eigenvalue counting functions.

Corollary 3.6. Let $t>0$ and $\varepsilon \in(0,1)$. For any $\lambda$ we have

$$
\mathrm{N}_{\Omega, t A}(\lambda) \geq \sum_{j \in J_{k}} \mathrm{~N}_{S^{k, j}, t A^{k, j}}\left((1-\varepsilon)\left(\lambda-\varepsilon^{-1} t^{2} \alpha_{k}^{2}\right)\right)
$$

and

$$
\begin{aligned}
\mathrm{N}_{\Omega, t A}(\lambda) \leq \mathrm{N}_{R_{\delta}^{k}, t A}\left(\lambda+\varepsilon^{-1} t^{2} \alpha_{k}^{2}+C_{3} 2^{2 k} \delta^{-2}\right) \\
+\sum_{j \in J_{k}} \mathrm{~N}_{S^{k, j}, t A^{k, j}}\left((1+\varepsilon)\left(\lambda+\varepsilon^{-1} t^{2} \alpha_{k}^{2}+C_{3} 2^{2 k} \delta^{-2}\right)\right) .
\end{aligned}
$$




\subsection{Proof of Theorem 1.1}

Write $\lambda(t)=(\Lambda+\gamma(t)) t$ with $\Lambda \in \mathbb{R}$ and $\gamma(t) \rightarrow 0$ as $t \rightarrow+\infty$. For $t>0, k \geq k_{0}$ and $\varepsilon, \delta, \rho \in(0,1)$ we can combine Corollary 3.6, Proposition 3.1, the homogeneity of $\nu^{+}$and (15) to get

$$
\begin{aligned}
\frac{1}{t} \mathrm{~N}_{\Omega, t A}(\lambda(t)) & \geq \sum_{j \in J_{k}} 2^{-2 k}(1-\rho)^{2} \nu^{+}\left(b^{k, j},(1-\varepsilon) \Gamma_{k}^{-}(t)\right) \\
& =(1-\rho)^{2} \int_{\Omega} \nu^{+}\left(B^{k},(1-\varepsilon) \Gamma_{k}^{-}(t)\right)
\end{aligned}
$$

with $\Gamma_{k}^{-}(t)=\Lambda+\gamma(t)-\varepsilon^{-1} t \alpha_{k}^{2}-C_{2}(1-\varepsilon)^{-1} t^{-1} 2^{2 k} \rho^{-2}$. Similarly

$$
\frac{1}{t} \mathrm{~N}_{\Omega, t A}(\lambda(t)) \leq \frac{1}{t} \mathrm{~N}_{R_{\delta}^{k}, t A}\left(t \Gamma_{k}^{+}(t)\right)+\int_{\Omega} \nu^{+}\left(B^{k},(1+\varepsilon) \Gamma_{k}^{+}(t)\right)
$$

with $\Gamma_{k}^{+}(t)=\Lambda+\gamma(t)+\varepsilon^{-1} t \alpha_{k}^{2}+C_{3} t^{-1} 2^{2 k} \delta^{-2}$.

Next recall that $\left(\beta_{k}\right)_{k \geq k_{0}}$ is a non-increasing positive sequence with $\beta_{k} \rightarrow 0$ as $k \rightarrow \infty$. Thus $\left(2^{2 k} \beta_{k}^{-1}\right)_{k \geq k_{0}}$ is an unbounded increasing sequence; it follows that we can define an unbounded non-decreasing function by setting

$$
k(t)=\min \left\{k \geq k_{0}: 2^{2 k} \beta_{k}^{-1} \geq t\right\}
$$

for any $t>0$. Note that, if $t>2^{2 k_{1}} \beta_{k_{1}}^{-1}$ then $t \in\left(2^{2 k(t)-2} \beta_{k(t)-1}^{-1}, 2^{2 k(t)} \beta_{k(t)}^{-1}\right]$ so

$$
t \alpha_{k(t)}^{2}=t 2^{-2 k(t)-3} \beta_{k(t)}^{2} \leq 2^{-3} \beta_{k(t)} \quad \text { and } \quad t^{-1} 2^{2 k(t)} \leq 4 \beta_{k(t)-1} .
$$

Hence $t \alpha_{k(t)}^{2}, t^{-1} 2^{2 k(t)} \rightarrow 0$ as $t \rightarrow \infty$. It follows that, for fixed $\varepsilon, \delta$ and $\rho$,

$$
\Gamma_{k(t)}^{-}(t), \Gamma_{k(t)}^{+}(t) \rightarrow \Lambda \text { as } t \rightarrow \infty .
$$

Now put $k=k(t)$ in (23). Lemma 3.4 and (25) then give

$$
\liminf _{t \rightarrow \infty} \frac{1}{t} \mathrm{~N}_{\Omega, t A}(\lambda(t)) \geq(1-\rho)^{2} \int_{\Omega} \nu^{-}(B,(1-\varepsilon) \Lambda)
$$

for any $\varepsilon, \rho \in(0,1)$. Taking $\varepsilon, \rho \rightarrow 0^{+}$(together with the Fatou-Lebesgue theorem and lower semi-continuity of $\nu^{-}$) now leads to the lower bound in Theorem 1.1.

To obtain the upper bound firstly apply Proposition 2.5 to get the bound

$$
\frac{1}{t} \mathrm{~N}_{R_{\delta}^{k(t)}, t A}\left(t \Gamma_{k(t)}^{+}(t)\right) \leq 2 C_{1}\left(\|B\|_{L_{\mathscr{A}}\left(R_{\delta}^{k(t)}\right)}+\left|\Gamma_{k(t)}^{+}(t)\right|\|1\|_{L_{\mathscr{A}}\left(R_{\delta}^{k(t)}\right)}\right) .
$$

Now $B, 1 \in L_{\mathscr{A}}(\Omega)$ while $(17)$ and (13) give $\left|R_{\delta}^{k(t)}\right| \leq\left|\Omega \backslash \overline{\Omega^{k(t)}}\right|+4|\Omega| \delta \rightarrow 4|\Omega| \delta$ as $t \rightarrow \infty$. Thus the right hand side of (26) must decay to 0 if we take $t \rightarrow \infty$ and then $\delta \rightarrow 0^{+}$. On the other hand Lemma 3.4 and (25) give

$$
\limsup _{t \rightarrow \infty} \int_{\Omega} \nu^{+}\left(B^{k(t)},(1+\varepsilon) \Gamma_{k(t)}^{+}(t)\right) \leq \int_{\Omega} \nu^{+}(B,(1+\varepsilon) \Lambda)
$$

for any $\varepsilon \in(0,1)$. The upper bound in Theorem 1.1 now follows it we put $k=k(t)$ in (24), take $t \rightarrow \infty$ and then take $\delta, \varepsilon \rightarrow 0^{+}$. 


\section{Approximate Zero Modes}

\subsection{Reduction to the disc}

Most of the work in establishing Theorem 1.2 lies in establishing a version of this result for single signed fields on $\mathbb{D}$, the (open) unit disc in $\mathbb{R}^{2}$. We firstly quote this as a separate result and then show how the more general result follows.

Theorem 4.1. Suppose $B \in C^{\alpha}(\overline{\mathbb{D}})$ for some $\alpha \in(0,1)$ and $B$ is single signed on $\mathbb{D}$. If $\lambda(t) \geq C e^{-c t^{\sigma}}$ for some constants $\sigma \in(0,1)$ and $c, C>0$ then

$$
\liminf _{t \rightarrow \infty} \frac{1}{t} \mathrm{~N}_{\mathbb{D}, t A}(\lambda(t)) \geq \Phi_{\mathbb{D}}(|B|) .
$$

Remark 4.1 (General discs). Let $R>0$ and suppose $B \in C^{\alpha}\left(\overline{\mathbb{D}}_{R}\right)$ is single signed and generated by the potential $A \in L_{\text {loc }}^{2}\left(\mathbb{D}_{R}\right)$. Setting $A^{\prime}(x)=R A(R x)$ defines a potential $A^{\prime} \in L_{\text {loc }}^{2}(\mathbb{D})$ with associated field given by $B^{\prime}(x)=R^{2} B(R x)$; in particular $B^{\prime} \in C^{\alpha}(\overline{\mathbb{D}})$ is single signed and $\Phi_{\mathbb{D}}\left(\left|B^{\prime}\right|\right)=\Phi_{\mathbb{D}_{R}}(|B|)$. On the other hand, the expression $\mathcal{U}_{R} u(x)=R u(R x)$ defines a unitary map $\mathcal{U}_{R}: L^{2}\left(\mathbb{D}_{R}\right) \rightarrow$ $L^{2}(\mathbb{D})$ with $\mathcal{U}_{R} \mathcal{P}_{\mathbb{D}_{R}, t A} \mathcal{U}_{R}^{*}=R^{-2} \mathcal{P}_{\mathbb{D}, t A^{\prime}}$. Thus $\mathrm{N}_{\mathbb{D}_{R}, t A}(\lambda)=\mathrm{N}_{\mathbb{D}, t A^{\prime}}\left(R^{2} \lambda\right)$ for any $\lambda$. It follows that Theorem 4.1 generalises to cover any disc in $\mathbb{R}^{2}$ (translation is clearly not an issue).

Proof of Theorem 1.2. Set $\Omega_{ \pm}=\left\{x \in \Omega_{0}: \pm B(x)>0\right\}$. Then $\Omega_{+} \cup \Omega_{-}$is open (as $B$ is continuous on $\Omega_{0}$ ), so the Vitali covering theorem (see [16], for example) allows us to find a countable sequence of mutually disjoint open discs $\mathbb{D}^{1}, \mathbb{D}^{2}, \cdots \subseteq \Omega_{+} \cup \Omega_{-}$with $\left|\left(\Omega_{+} \cup \Omega_{-}\right) \backslash \Omega_{\mathbb{D}}\right|=0$ where $\Omega_{\mathbb{D}}=\cup_{k \in \mathbb{N}} \mathbb{D}^{k}$. Since $B$ is continuous and non-zero on $\Omega_{+} \cup \Omega_{-}$it must be single signed on each $\mathbb{D}^{k}$. A straightforward variational argument also shows $N_{\Omega, t A}(\lambda) \geq \sum_{k \in \mathbb{N}} N_{\mathbb{D}^{k}, t A}(\lambda)$ for any $\lambda$. If $\lambda(t) \geq C e^{-c t^{\sigma}}$ we can then apply Theorem 4.1 (see also Remark 4.1) and the superadditivity of lim inf to get

$$
\liminf _{t \rightarrow \infty} \frac{1}{t} \mathrm{~N}_{\Omega, t A}(\lambda(t)) \geq \sum_{k \in \mathbb{N}} \Phi_{\mathbb{D}^{k}}(|B|)=\Phi_{\Omega_{\mathbb{D}}}(|B|) .
$$

However $\Phi_{\Omega_{\mathbb{D}}}(|B|)=\Phi_{\Omega}(|B|)$ since $\left|\Omega \backslash \Omega_{0}\right|=\left|\left(\Omega_{+} \cup \Omega_{-}\right) \backslash \Omega_{\mathbb{D}}\right|=0$ while $B=0$ on $\Omega_{0} \backslash\left(\Omega_{+} \cup \Omega_{-}\right)$.

\subsection{Reduction to the circle}

From Remark 2.3 it suffices to prove Theorem 4.1 in the case that $B$ is nonnegative. Clearly we can also impose the flux normalisation condition

$$
\Phi_{\mathbb{D}}(|B|)=\Phi_{\mathbb{D}}(B)=\frac{1}{2 \pi} \int_{\mathbb{D}} B=1
$$

(note that, Theorem 4.1 holds trivially when $B \equiv 0$ ). We will henceforth assume $B \in C^{\alpha}(\overline{\mathbb{D}})$ is non-negative on $\mathbb{D}$ and satisfies $(28)$. Let

$$
\beta=\|B\|_{L^{\infty}(\mathbb{D})}
$$


Since $\mathbb{D}$ is simply connected Proposition 2.7 gives us the freedom to choose any magnetic potential $A \in L_{\text {loc }}^{2}(\mathbb{D})$ whose associated field is $B$. A convenient choice can be made via the "scalar potential". Firstly let $\phi: \mathbb{D} \rightarrow \mathbb{R}$ be the solution of $\Delta \phi=B$ on $\mathbb{D}$, with $\phi=0$ on $\partial \mathbb{D}=S^{1}$; such a solution exists, is unique and satisfies $\phi \in C^{2, \alpha}(\overline{\mathbb{D}})$ (see [13]). If we set $A=\left(-\nabla_{2} \phi, \nabla_{1} \phi\right) \in C^{1, \alpha}\left(\overline{\mathbb{D}}, \mathbb{R}^{2}\right)$ then $A$ is a magnetic potential with associated field curl $A=\nabla_{1}^{2} \phi+\nabla_{2}^{2} \phi=B$. Furthermore $\mp i \nabla_{ \pm} \phi=\nabla_{2} \phi \mp i \nabla_{1} \phi=-\left(A_{1} \pm i A_{2}\right)$ so

$$
-i e^{\mp t \phi} \nabla_{ \pm}\left(e^{ \pm t \phi} \cdot\right)=-i \nabla_{ \pm} \mp i t \nabla_{ \pm} \phi=P_{t A, \pm} .
$$

For $u \in C_{0}^{2}\left(\mathbb{D}, \mathbb{C}^{2}\right)$ it follows that

$$
\mathbf{p}_{\mathbb{D}, t A}(u)=\left\|e^{-t \phi} \nabla_{+}\left(u_{+} e^{t \phi}\right)\right\|^{2}+\left\|e^{t \phi} \nabla_{-}\left(u_{-} e^{-t \phi}\right)\right\|^{2} .
$$

Setting $v_{ \pm}=u_{ \pm} e^{ \pm t \phi}$ we have $u \in C_{0}^{2}\left(\mathbb{D}, \mathbb{C}^{2}\right)$ iff $v \in C_{0}^{2}\left(\mathbb{D}, \mathbb{C}^{2}\right)$, while

$$
\|u\|^{2}=\int_{\mathbb{D}}\left|v_{+}\right|^{2} e^{-2 t \phi}+\int_{\mathbb{D}}\left|v_{-}\right|^{2} e^{2 t \phi}
$$

and

$$
\mathbf{p}_{\mathbb{D}, t A}(u)=\int_{\mathbb{D}}\left|\nabla_{+} v_{+}\right|^{2} e^{-2 t \phi}+\int_{\mathbb{D}}\left|\nabla_{-} v_{-}\right|^{2} e^{2 t \phi} .
$$

It is straightforward to check that $C_{0}^{2}\left(\mathbb{D}, \mathbb{C}^{2}\right)$ is a core for the form $\mathbf{p}_{\mathbb{D}, t A}$.

Using a variational argument we can establish Theorem 4.1 by constructing sufficiently large spaces of test functions $\mathcal{X}_{t} \subset C_{0}^{2}\left(\mathbb{D}, \mathbb{C}^{2}\right)$ for which

$$
\mathbf{p}_{\mathbb{D}, t A}(u) \leq \lambda(t)\|u\|^{2}, \quad u \in \mathcal{X}_{t} .
$$

By the strong maximum principle (see [13], for example) $\phi$ is strictly negative on D. As $\lambda(t) \ll 1$ the exponential weights in $(31)$ and $(32)$ then encourage us to seek test functions with $v_{-}=0$ and $\nabla_{+} v_{+}=0$, at least away from the boundary $\partial \mathbb{D}=S^{1}$. Identifying $\mathbb{R}^{2}$ with $\mathbb{C}$ in the standard way we have $\nabla_{+}=2 \bar{\partial}$, so $\nabla_{+} v_{+}=0$ iff $v_{+}$is in $\mathcal{O}(\mathbb{D})$ the set of holomorphic functions on $\mathbb{D}$. To get an element of $C_{0}^{2}(\mathbb{D})$ we multiply by a cut-off function $\chi \in C_{0}^{\infty}(\mathbb{D})$ (which should be $\mathbb{R}$-valued and differ from 1 only near $\left.S^{1}\right)$. If we take $v_{+} \in \mathcal{O}(\mathbb{D})$ and set

$$
u=\left(\begin{array}{c}
\chi v_{+} e^{-t \phi} \\
0
\end{array}\right) \in C_{0}^{2}\left(\mathbb{D}, \mathbb{C}^{2}\right)
$$

then $\nabla_{+}\left(\chi v_{+}\right)=v_{+} \nabla_{+} \chi$ and $\left|\nabla_{+} \chi\right|^{2}=|\nabla \chi|^{2}$, so (31) and (32) become

$$
\|u\|^{2}=\int_{\mathbb{D}}|\chi|^{2}\left|v_{+}\right|^{2} e^{-2 t \phi}
$$

and

$$
\mathbf{p}_{\mathbb{D}, t A}(u)=\int_{\mathbb{D}}|\nabla \chi|^{2}\left|v_{+}\right|^{2} e^{-2 t \phi} .
$$

The remainder of our analysis will be focused near the boundary of $\mathbb{D}$ (on a neighbourhood of where $\nabla \chi \neq 0$ ). The information we need about $B$ is captured 
by the boundary behaviour of $\phi$. Let $h$ denote the outward normal derivative of $\phi$ on $\partial \mathbb{D}=S^{1}$. Using polar coordinates $(r, \theta)$ on $\overline{\mathbb{D}}$ we have $h(\theta)=\nabla_{r} \phi(1, \theta)$, while $h \in C^{1, \alpha}\left(S^{1}\right)$ since $\phi \in C^{2, \alpha}(\overline{\mathbb{D}})$. As a consequence of the maximum principle $h$ is strictly positive (see [13, Lemma 3.4$]$ ); the quantity

$$
\kappa=\max \left\{\|h\|_{L^{\infty}\left(S^{1}\right)},\|1 / h\|_{L^{\infty}\left(S^{1}\right)}\right\}
$$

is thus finite and positive. The divergence theorem and condition (28) also give

$$
\int_{S^{1}} h=\int_{\partial \mathbb{D}} \nabla_{r} \phi=\int_{\mathbb{D}} \operatorname{div} \nabla \phi=\int_{\mathbb{D}} B=2 \pi .
$$

Let $H^{2}\left(S^{1}\right)$ denote the Hardy space on $S^{1}$. Each $f \in H^{2}\left(S^{1}\right)$ is the boundary trace of a unique function $E f \in \mathcal{O}(\mathbb{D})$ ( $E$ is just the usual identification of $H^{2}\left(S^{1}\right)$ with the Hardy space on $\mathbb{D})$. Now let $f \in H^{2}\left(S^{1}\right)$ and set $v_{+}=E f$. Using polar coordinates on $\overline{\mathbb{D}}$ define a function $w_{f}:[0,1] \rightarrow[0, \infty)$ by

$$
w_{f}(r)=\int_{0}^{2 \pi}\left|v_{+}(r, \theta)\right|^{2} e^{-2 t \phi(r, \theta)} d \theta .
$$

When $f \neq 0$ we can then set

$$
\omega_{f}=\left.\nabla_{r} \log \left(r w_{f}(r)\right)\right|_{r=1}=\frac{\nabla_{r} w_{f}(1)}{w_{f}(1)}+1 .
$$

To create a test function we still need to fix the cut-off function $\chi$. We want this to be radial (for convenience) and decaying in a layer of width $\delta>0$ near the boundary of $\mathbb{D}$. Choose a smooth non-decreasing function $\rho: \mathbb{R} \rightarrow \mathbb{R}$ with $\rho=0$ on $(-\infty, 0], \rho=1$ on $[1, \infty)$, and $|\nabla \rho| \leq 1 / \sqrt{2}$. For $\delta \in(0,1)$ and $r \in[0,1]$ set

$$
\rho_{\delta}(r)=\rho\left(\delta^{-1}(1-r)\right)
$$

Proposition 4.2. Suppose $0 \neq f \in H^{2}\left(S^{1}\right)$ satisfies $\omega_{f} \leq-6 \beta t \delta$ with $t>0$ and $\delta \in(0,1 / 3]$. Let $u$ be given by (33) where $v_{+}=E f$ and $\chi(r, \theta)=\rho_{\delta}(r)$. Then

$$
\mathbf{p}_{\mathbb{D}, t A}(u) \leq \frac{1}{4 \delta^{2}} \exp \left[\omega_{f} \delta+6 \beta t \delta^{2}\right]\|u\|^{2} .
$$

To use this estimate we need further information on the behaviour of $\omega_{f}$. A summary of the necessary information is contained in the next result.

Proposition 4.3. Suppose $\nu_{t}>0$ for $t \geq 1$. Then there exist constants $C_{4,1}, C_{4,2}$ and spaces $X_{t} \subset H^{2}\left(S^{1}\right)$ for $t \geq 1$ such that $\omega_{f} \leq-\nu_{t}$ for all $0 \neq f \in X_{t}$ and

$$
\operatorname{dim} X_{t} \geq t-C_{4,1} \nu_{t}-C_{4,2} t^{(1-2 \alpha)_{+}} .
$$

Propositions 4.2 and 4.3 are proved at the end of Sections 4.3 and 4.4 respectively. 
Remark 4.2. The test functions given by (33) are purely spin-up (only the component $u_{+}$is non-zero). To deal with the case $B \leq 0$ directly by an argument similar to that above we would need to consider purely spin-down test functions of the form

$$
u=\left(\begin{array}{c}
0 \\
\chi v_{-} e^{t \phi}
\end{array}\right)
$$

where $v_{-} \in \overline{\mathcal{O}}(\mathbb{D})$ (the set of anti-holomorphic functions on $\mathbb{D}$ ). Clearly the antilinear isometry $\mathcal{J}$ from Remark 2.3 sends test functions of one type to the other.

Proof of Theorem 4.1. Choose $C_{5,1}$ and $C_{5,2}$ so that

$$
t^{1-\sigma} e^{-c t^{\sigma}} \leq 4 C C_{5,1}^{2} \quad \text { and } \quad C_{5,1}\left(C_{5,2}-6 \beta C_{5,1}\right)=2 c
$$

for all $t \geq 0$. Set $\delta_{t}=C_{5,1} t^{(\sigma-1) / 2}$ and $\nu_{t}=C_{5,2} t^{(\sigma+1) / 2}$. Suppose $t \geq t_{0}$, where $t_{0}=\max \left\{1,\left(3 C_{5,1}\right)^{2 /(1-\sigma)}\right\}$; thus $t \geq 1$ and $\delta_{t} \in(0,1 / 3]$. Let $X_{t} \subset H^{2}\left(S^{1}\right)$ be as given by Proposition 4.3 and set

$$
\mathcal{X}_{t}=\left\{\left(\begin{array}{c}
\rho_{\delta_{t}}(E f) e^{-t \phi} \\
0
\end{array}\right): f \in X_{t}\right\} \subset C_{0}^{2}\left(\mathbb{D}, \mathbb{C}^{2}\right) .
$$

Since $E f=0$ iff $f=0$ we get

$$
\operatorname{dim} \mathcal{X}_{t}=\operatorname{dim} X_{t} \geq t-C_{4,1} C_{5,2} t^{(\sigma+1) / 2}-C_{4,2} t^{(1-2 \alpha)_{+}},
$$

so $\liminf \operatorname{in}_{t \rightarrow \infty} t^{-1} \operatorname{dim} \mathcal{X}_{t}=1$.

Let $0 \neq f \in X_{t}$ and set $u=\left(\begin{array}{c}\rho_{\delta_{t}}(E f) e^{-t \phi} \\ 0\end{array}\right) \in \mathcal{X}_{t}$. Now

$\nu_{t} \delta_{t}-6 \beta t \delta_{t}^{2}=C_{5,1} C_{5,2} t^{\sigma}-6 \beta C_{5,1}^{2} t^{\sigma}=2 c t^{\sigma} \quad$ and $\quad \frac{1}{4 \delta_{t}^{2}} e^{-c t^{\sigma}}=\frac{t^{1-\sigma}}{4 C_{5,1}^{2}} e^{-c t^{\sigma}} \leq C$

by (40), so Proposition 4.3 leads to $\omega_{f} \leq-\nu_{t} \leq-6 \beta t \delta_{t}$. Proposition 4.2 then gives

$$
\mathbf{p}_{\mathbb{D}, t A}(u) \leq \frac{1}{4 \delta_{t}^{2}} \exp \left[-\nu_{t} \delta_{t}+6 \beta t \delta_{t}^{2}\right]\|u\|^{2} \leq C e^{-c t^{\sigma}}\|u\|^{2}
$$

The result now follows from a variational argument.

Remark 4.3. A more precise version of (the error term in) (27) can be obtained from (41); setting $C_{5,3}=\max \left\{1,3 C_{5,1}, C_{4,1} C_{5,2}\right\}$ we get

$$
\mathrm{N}_{\mathbb{D}, t A}(\lambda(t)) \geq t-C_{5,3} t^{(\sigma+1) / 2}-C_{4,2} t^{(1-2 \alpha)_{+}}
$$

for all $t \geq 0$ (note that, the right hand side is negative when $t<t_{0}$ ).

Remark 4.4. With some modifications to the proof we can extend Theorem 4.1 to obtain lower bounds for $\mathrm{N}_{\mathbb{D}, t A}(\varepsilon)$ for fixed $\varepsilon>0$. With $C_{6,1}=2 \sqrt{\varepsilon}$ and $C_{6,2}=6 \beta C_{6,1}^{-1}$ set $\delta_{t}=C_{6,1}^{-1} t^{-1 / 2}$ and $\nu_{t}=\left(C_{6,1} \log t+C_{6,2}\right) t^{1 / 2}$ for $t \geq 0$. 
Suppose $t \geq t_{0}$, where $t_{0}=\max \left\{1,9 C_{6,1}^{-2}\right\}$; thus $t \geq 1$ and $\delta_{t} \in(0,1 / 3]$. Let $X_{t}$ and $\mathcal{X}_{t}$ be as above. Now

$\nu_{t} \delta_{t}-6 \beta t \delta_{t}^{2}=C_{6,1} C_{6,1}^{-1} \log t+C_{6,2} C_{6,1}^{-1}-6 \beta C_{6,1}^{-2}=\log t \quad$ and $\quad \frac{1}{4 \delta_{t}^{2}}=\frac{C_{6,1}^{2} t}{4}=\varepsilon t$,

so the middle estimate in (42) gives $\mathbf{p}_{\mathbb{D}, t A}(u) \leq \varepsilon\|u\|^{2}$ for any $u \in \mathcal{X}_{t}$. On the other hand

$$
\operatorname{dim} \mathcal{X}_{t}=\operatorname{dim} X_{t} \geq t-C_{4,1}\left(C_{6,1} \log t+C_{6,2}\right) t^{1 / 2}-C_{4,2} t^{(1-2 \alpha)_{+}}
$$

for any $t \geq t_{0}$. Setting $C_{6,3}=\max \left\{1, C_{4,1}\left(C_{6,1}+C_{6,2}\right), 3 / C_{6,1}\right\}$ we then obtain

$$
\mathrm{N}_{\mathbb{D}, t A}(\varepsilon) \geq t-C_{6,3} t^{1 / 2} \log (t+2)-C_{4,2} t^{(1-2 \alpha)_{+}}
$$

for all $t \geq 0$ (note that, the right hand side is negative when $t<t_{0}$ ).

It is possible that the log is an artefact of our method and the second term in the asymptotics of $\mathrm{N}_{\mathbb{D}, t A}(\varepsilon)$ should be $O\left(t^{1 / 2}\right)$, at least for sufficiently regular $B$.

\subsection{Quadratic form estimates}

The aim of this section is to prove Proposition 4.2. The presentation is simplified if we switch to polar coordinates; the magnetic momentum operators are then

$$
P_{r}=-i \nabla_{r}-t A_{r}=-i \nabla_{r}+\frac{t}{r} \nabla_{\theta} \phi \quad \text { and } \quad P_{\theta}=-\frac{i}{r} \nabla_{\theta}-t A_{\theta}=-\frac{i}{r} \nabla_{\theta}-t \nabla_{r} \phi .
$$

For functions $u, v$ defined on $\overline{\mathbb{D}}$ we will use $\langle u, v\rangle_{S^{1}}$ and $\|u\|_{S^{1}}$ to indicate the $L^{2}$-inner product and norm in the $S^{1}$ variable only; that is,

$$
\langle u, v\rangle_{S^{1}}=\int_{0}^{2 \pi} \overline{u(r, \theta)} v(r, \theta) d \theta \quad \text { and } \quad\|u\|_{S^{1}}^{2}=\int_{0}^{2 \pi}|u(r, \theta)|^{2} d \theta
$$

which depend on $r \in[0,1]$.

Firstly we take a more detailed look at the function $w_{f}$ defined in (38).

Lemma 4.4. Let $f \in H^{2}\left(S^{1}\right)$ and set $u=(E f) e^{-t \phi}$. Then

$$
\nabla_{r} w_{f}=2\left\langle u, P_{\theta} u\right\rangle_{S^{1}} \quad \text { and } \quad \nabla_{r}\left(r \nabla_{r} w_{f}\right)=4 r\left\|P_{\theta} u\right\|_{S^{1}}^{2}-2 \operatorname{tr}\langle u, B u\rangle_{S^{1}} .
$$

Proof. Firstly observe that for any functions $v, w$ on $\overline{\mathbb{D}}$ we have

$$
\begin{aligned}
\nabla_{r}\langle v, w\rangle_{S^{1}} & =\left\langle i P_{r} v, w\right\rangle_{S^{1}}+\left\langle v, i P_{r} w\right\rangle_{S^{1}} \\
& =\left\langle P_{\theta} v, w\right\rangle_{S^{1}}+\left\langle v, P_{\theta} w\right\rangle_{S^{1}}-i\langle Q v, w\rangle_{S^{1}}+i\langle v, Q w\rangle_{S^{1}},
\end{aligned}
$$

where $Q=P_{r}+i P_{\theta}=e^{-i \theta} P_{t A,+}$. The second expression for $Q$ and (30) lead to

$$
Q u=-i e^{-i \theta} e^{-t \phi}\left(\nabla_{+}(E f)\right)=0
$$


(recall that $E f \in \mathcal{O}(\mathbb{D}))$. Now $w_{f}=\|u\|_{S^{1}}^{2}$ so $(43)$ and $(44)$ give

$$
\nabla_{r} w_{f}=\left\langle P_{\theta} u, u\right\rangle_{S^{1}}+\left\langle u, P_{\theta} u\right\rangle_{S^{1}}=2\left\langle u, P_{\theta} u\right\rangle_{S^{1}}
$$

as $P_{\theta}$ is symmetric with respect to $\langle\cdot, \cdot\rangle_{S^{1}}$. Using (43) and (44) again then gives

$$
\begin{aligned}
& \nabla_{r}\left(r \nabla_{r} w_{f}\right) \\
& \quad=2\left\langle P_{\theta} u, r P_{\theta} u\right\rangle_{S^{1}}+2\left\langle u, P_{\theta}\left(r P_{\theta} u\right)\right\rangle_{S^{1}}-2 i\left\langle Q u, r P_{\theta} u\right\rangle_{S^{1}}+2 i\left\langle u, Q\left(r P_{\theta} u\right)\right\rangle_{S^{1}} \\
& \quad=4 r\left\|P_{\theta} u\right\|_{S^{1}}^{2}+2 i\left\langle u,\left[Q, r P_{\theta}\right] u\right\rangle_{S^{1}} .
\end{aligned}
$$

However $\left[Q, r P_{\theta}\right]=\left[P_{r}, r P_{\theta}\right]=-i \nabla_{r}\left(-r t A_{\theta}\right)+i \nabla_{\theta}\left(-t A_{r}\right)=i t r B$.

The formulae for the derivatives of $w_{f}$ given by Lemma 4.4 lead to the following.

Lemma 4.5. Let $0 \leq r_{0} \leq 1$ and $f \in H^{2}\left(S^{1}\right)$. If $0 \leq b \leq-\left(\omega_{f}+2 \beta t\left(1-r_{0}\right)\right)$ then $e^{b r} r w_{f}(r)$ is decreasing for $r \in\left[r_{0}, 1\right]$.

Proof. For any $b \in \mathbb{R}$ set $\ell_{b}(r)=\log \left(e^{b r} r w_{f}(r)\right)=\log \left(w_{f}(r)\right)+\log r+b r$. Then

$$
\begin{aligned}
\nabla_{r}\left(r \nabla_{r} \ell_{b}\right) & =\frac{\nabla_{r}\left(r \nabla_{r} w_{f}\right)}{w_{f}}-r \frac{\left(\nabla_{r} w_{f}\right)^{2}}{w_{f}^{2}}+b \\
& =\frac{r}{w_{f}^{2}}\left[4\left\|P_{\theta} u\right\|_{S^{1}}^{2}\|u\|_{S^{1}}^{2}-4\left\langle u, P_{\theta} u\right\rangle_{S^{1}}^{2}\right]-2 \operatorname{tr} \frac{\langle u, B u\rangle_{S^{1}}}{\|u\|_{S^{1}}^{2}}+b
\end{aligned}
$$

using Lemma 4.4 and the fact that $w_{f}=\|u\|_{S^{1}}^{2}$. Now $[\cdot] \geq 0,\langle u, B u\rangle_{S^{1}} \leq \beta\|u\|_{S^{1}}^{2}$ (recall (29)) and $r \leq 1$. Hence $\nabla_{r}\left(r \nabla_{r} \ell_{b}\right) \geq-2 \beta t+b$. Integrating from $r$ to 1 and using the fact that $\nabla_{r} \ell_{b}(1)=\omega_{f}+b$ we get

$$
r \nabla_{r} \ell_{b}(r) \leq \nabla_{r} \ell_{b}(1)+\int_{r}^{1}(2 \beta t-b) d r=\omega_{f}+2 \beta t(1-r)+b r .
$$

If $r_{0} \leq r \leq 1$ and $0 \leq b \leq-\left(\omega_{f}+2 \beta t\left(1-r_{0}\right)\right)$ it follows that the right hand side is non-positive, and hence $\nabla_{r} \ell_{b}(r) \leq 0$. Thus $\exp \left(\ell_{b}(r)\right)=e^{b r} r w_{f}(r)$ is decreasing for $r \in\left[r_{0}, 1\right]$.

Proof of Proposition 4.2. Set $r_{\delta}=1-\delta$ and $b=-\left(\omega_{f}+6 \beta t \delta\right) \geq 0$, so $\rho_{\delta}(r)=1$ for $r \leq r_{\delta}$ while $e^{b r} r w_{f}(r)$ is decreasing for $r \in[1-3 \delta, 1]$ by Lemma 4.5. Using (34) and (38) we then get

$$
\begin{aligned}
\|u\|^{2}= & \int_{0}^{1} \rho_{\delta}(r)^{2} r w_{f}(r) d r \geq \int_{1-3 \delta}^{r_{\delta}} r w_{f}(r) d r \\
& \geq r_{\delta} w_{f}\left(r_{\delta}\right) \int_{1-3 \delta}^{r_{\delta}} e^{b\left(r_{\delta}-r\right)} d r=r_{\delta} w_{f}\left(r_{\delta}\right) \frac{1}{b}\left(e^{2 b \delta}-1\right) \geq r_{\delta} w_{f}\left(r_{\delta}\right) 2 \delta e^{b \delta} .
\end{aligned}
$$

To estimate $\mathbf{p}_{\mathbb{D}, t A}(u)$ note that $|\nabla \chi|^{2}=\left|\nabla \rho_{\delta}\right|^{2} \leq 1 /\left(2 \delta^{2}\right)$ and $\operatorname{supp}\left(\nabla \rho_{\delta}\right) \subseteq$ $\left[r_{\delta}, 1\right]$, while $r w_{f}(r)$ is decreasing for $r \in\left[r_{\delta}, 1\right] \subset[1-3 \delta, 1]$ by Lemma 4.5. Thus (35) and (38) lead to

$$
\mathbf{p}_{\mathbb{D}, t A}(u)=\int_{0}^{1}\left|\nabla \rho_{\delta}(r)\right|^{2} r w_{f}(r) d r \leq \frac{1}{2 \delta^{2}} \int_{r_{\delta}}^{1} r w_{f}(r) d r \leq \frac{1}{2 \delta} r_{\delta} w_{f}\left(r_{\delta}\right) .
$$

Combined with the previous estimate we then get $\mathbf{p}_{\mathbb{D}, t A}(u) \leq(2 \delta)^{-2} e^{-b \delta}\|u\|^{2}$. 


\subsection{Analysis on the circle}

Let $f \in H^{2}\left(S^{1}\right)$ and set $u=(E f) e^{-t \phi}$. Then $u(1, \theta)=f(\theta)$ so $w_{f}(1)=\|f\|^{2}$. Also $A_{\theta}(1, \theta)=\nabla_{r} \phi(1, \theta)=h(\theta)$. Introducing the operator $T=-i \nabla-t h$ on $S^{1}$, Lemma 4.4 now gives $\nabla_{r} w_{f}(1)=2\langle f, T f\rangle$. If $f \neq 0$ it follows that

$$
\omega_{f}=2 \frac{\langle f, T f\rangle}{\|f\|^{2}}+1
$$

To construct the space $X_{t}$ in Proposition 4.3 we need to find $f \in H^{2}\left(S^{1}\right)$ for which $\omega_{f}$ is negative. In view of (46) this leads us to consider the spectral properties of the operator $T$ on $H^{2}\left(S^{1}\right)$. We begin by considering $T$ as an operator on $L^{2}\left(S^{1}\right)$ where a more explicit description is possible. Set

$$
\eta(\theta)=\int_{0}^{\theta} h(\omega) d \omega
$$

so $\eta(0)=0, \eta(2 \pi)=2 \pi($ recall $(37))$ and $\nabla \eta=h$. Since $h$ and $1 / h$ are both continuous and bounded away from 0 it follows that $\eta$ is a $C^{1}$-diffeomorphism of $S^{1}$. Thus $\mathcal{U} f=f \circ \eta$ defines a unitary map $\mathcal{U}$ from $L^{2}\left(S^{1}\right)$ with its usual inner-product to $L^{2}\left(S^{1}\right)$ with weighted inner-product $\langle\cdot, \cdot\rangle_{h}$ given by $\langle f, g\rangle_{h}=\langle f, h g\rangle=\langle h f, g\rangle$. Using $\|\cdot\|_{h}$ to denote the corresponding norm we have

$$
\kappa^{-1}\|f\|^{2} \leq\|f\|_{h}^{2} \leq \kappa\|f\|^{2}
$$

for any $f \in L^{2}\left(S^{1}\right)$ (recall (36)). The image of the standard Fourier basis under $\mathcal{U}$ is $\left\{\xi_{n}: n \in \mathbb{Z}\right\}$ where $\xi_{n}=e^{i n \eta} / \sqrt{2 \pi}$ for $n \in \mathbb{Z}$. In particular, any $f \in L^{2}\left(S^{1}\right)$ can be written as $f=\sum_{n \in \mathbb{Z}} \gamma_{n} \xi_{n}$ for some constants $\gamma_{n}$ (given by $\gamma_{n}=\left\langle\xi_{n}, f\right\rangle_{h}$ ), whereupon $\|f\|_{h}^{2}=\sum_{n \in \mathbb{Z}}\left|\gamma_{n}\right|^{2}$. Since $-i \nabla \xi_{n}=n h \xi_{n}$ we get

$$
T \xi_{n}=(n-t) h \xi_{n}
$$

and thus

$$
\langle f, T f\rangle=\sum_{n \in \mathbb{Z}}(n-t)\left|\gamma_{n}\right|^{2} .
$$

For $M \geq 0$ let $Q_{M}$ denote the $\langle\cdot, \cdot\rangle_{h}$-orthogonal projection onto $\overline{\operatorname{Sp}}\left\{\xi_{n}: n>M\right\}$.

Lemma 4.6. Let $f \in L^{2}\left(S^{1}\right)$ and $M \geq 0$. Then

$$
\langle f, T f\rangle \leq(M-t)\|f\|_{h}^{2}+\left\langle Q_{M} f, T Q_{M} f\right\rangle .
$$

Proof. Write $f=\sum_{n \in \mathbb{Z}} \gamma_{n} \xi_{n}$ for some $\gamma_{n}$. Then $Q_{M} f=\sum_{n>M} \gamma_{n} \xi_{n}$ so

$$
\langle f, T f\rangle-\left\langle Q_{M} f, T Q_{M} f\right\rangle=\sum_{n \leq M}(n-t)\left|\gamma_{n}\right|^{2} \leq(M-t) \sum_{n \in \mathbb{Z}}\left|\gamma_{n}\right|^{2}=(M-t)\|f\|_{h}^{2}
$$

with the help of (49). 
We shall now move our attention to consider $T$ acting on $H^{2}\left(S^{1}\right)$. Let $\Pi^{+}$ denote the orthogonal projection of $L^{2}\left(S^{1}\right)$ onto $H^{2}\left(S^{1}\right)$ and $\Pi^{-}$its complement; that is

$$
\Pi^{+} f=\frac{1}{\sqrt{2 \pi}} \sum_{k \geq 0} \widehat{f}(k) e^{i k \theta} \quad \text { and } \quad \Pi^{-}=I-\Pi^{+},
$$

where $\widehat{f}(k)$ denotes the $k$ th Fourier coefficient of $f$. A key idea in our argument is the fact that, for large $n, \xi_{n}$ and $h \xi_{n}$ "almost" lie in the space $H^{2}\left(S^{1}\right)$ in the sense that $\Pi^{-} \xi_{n}$ and $\Pi^{-} h \xi_{n}$ become small. This is made more precise via the quantities

$$
\alpha_{m}=\sum_{n>m}\left\|\Pi^{-} \xi_{n}\right\|^{2} \quad \text { and } \quad \beta_{m}=\sum_{n>m}\left\|\Pi^{-} h \xi_{n}\right\|^{2} .
$$

Proposition 4.7. There exists a constant $C_{7}$ such that

$$
\alpha_{m}, \beta_{m} \leq C_{7}(m+1)^{-2 \alpha}, \quad m \geq 0 .
$$

We shall consider families of diffeomorphisms of $S^{1}$ which are related to $\eta$. Firstly note that a positively oriented homeomorphism of $S^{1}$ can be viewed as a continuous strictly increasing map $\psi: \mathbb{R} \rightarrow \mathbb{R}$ which satisfies $\psi(\theta+2 \pi)=\psi(\theta)+2 \pi$. If $\psi$ is differentiable then $\nabla \psi: \mathbb{R} \rightarrow \mathbb{R}$ is $2 \pi$-periodic and hence can be viewed as a map on $S^{1}$. It is straightforward to check that $\psi$ is a (positively oriented) $C^{2, \alpha}$ diffeomorphism of $S^{1}$ if $\nabla \psi \in C^{1, \alpha}\left(S^{1}\right)$ and $\nabla \psi$ is strictly positive; in this case $\nabla \psi$ and $\nabla \psi^{-1}$ are both uniformly bounded away from 0 , while $\nabla \psi^{-1} \in C^{1, \alpha}\left(S^{1}\right)$.

Proof of Proposition 4.7. For each $\tau \in[0,1]$ set $\eta_{\tau}(\theta)=\tau \eta(\theta)+(1-\tau) \theta$. Then $\eta_{\tau}(0)=0, \eta_{\tau}(2 \pi)=2 \pi$ and $\nabla \eta_{\tau}=\tau h+(1-\tau)$. Thus $\nabla \eta_{\tau} \in C^{1, \alpha}\left(S^{1}\right)$ with $\nabla \eta_{\tau} \geq \tau \kappa^{-1}+(1-\tau) \geq \kappa^{-1}$, so $\nabla \eta_{\tau}$ is bounded away from 0 uniformly in $\tau$. It follows that $\eta_{\tau}$ is a $C^{2, \alpha}$-diffeomorphism of $S^{1}$. Setting

$$
y_{\tau}=\frac{1}{\sqrt{2 \pi}} \nabla \eta_{\tau}^{-1}=\frac{1}{\sqrt{2 \pi}} \frac{1}{\left(\nabla \eta_{\tau}\right) \circ \eta_{\tau}^{-1}}
$$

we get $y_{\tau} \in C^{1, \alpha}\left(S^{1}\right)$, while $\left\|y_{\tau}\right\|_{C^{1, \alpha}\left(S^{1}\right)}$ can be bounded uniformly for $\tau \in[0,1]$. Using standard estimates for the Fourier coefficients of functions in $C^{1, \alpha}\left(S^{1}\right)$ (see [18], for example) we can then find $C_{7,1}$ so that

$$
\left|\widehat{y_{\tau}}(n)\right| \leq C_{7,1}|n|^{-1-\alpha}, \quad \tau \in[0,1], n \neq 0 .
$$

Now suppose $n \geq 0$ and $k>0$. Set $\tau=n /(k+n) \in[0,1]$. Then

$$
\begin{gathered}
\widehat{\xi_{n}}(-k)=\frac{1}{2 \pi} \int_{0}^{2 \pi} e^{i n \eta(\theta)+i k \theta} d \theta=\frac{1}{2 \pi} \int_{0}^{2 \pi} \frac{1}{\nabla \eta_{\tau}(\theta)} e^{i(k+n) \eta_{\tau}(\theta)} \nabla \eta_{\tau}(\theta) d \theta \\
=\frac{1}{\sqrt{2 \pi}} \int_{0}^{2 \pi} y_{\tau}(\omega) e^{i(k+n) \omega} d \omega=\widehat{y_{\tau}}(-(k+n)) .
\end{gathered}
$$

For any $m \geq 0$ we can now combine this with (50) to get

$$
\alpha_{m}=\sum_{n>m} \sum_{k>0}\left|\widehat{\xi_{n}}(-k)\right|^{2} \leq C_{7,1}^{2} \sum_{n>m} \sum_{k>0}(k+n)^{-2-2 \alpha} \leq \frac{C_{7,1}^{2}}{2 \alpha}(m+1)^{-2 \alpha} .
$$


We can estimate $\beta_{m}$ using a similar argument. In particular, we need to consider the $C^{2, \alpha}$-diffeomorphisms of $S^{1}$ given by $\zeta_{\tau}(\theta)=\tau \eta^{-1}(\theta)+(1-\tau) \theta, \tau \in[0,1]$. Then

$$
z_{\tau}=\frac{1}{\sqrt{2 \pi}} \nabla \zeta_{\tau}^{-1}
$$

is uniformly bounded in $C^{1, \alpha}\left(S^{1}\right)$, while for any $n \geq 0$ and $k>0$ it can be shown that the $(-k)$ th Fourier coefficient of $h \xi_{n}$ is just $\widehat{z_{\tau}}(-(k+n))$. The remainder of the argument to estimate $\beta_{m}$ proceeds exactly as for $\alpha_{m}$.

Proposition 4.7 establishes that $\alpha_{0}$ is finite; it follows that $\alpha_{m}$ is non-increasing with $\lim _{m \rightarrow \infty} \alpha_{m}=0$. A similar comment applies to $\beta_{m}$. Also recall that $Q_{m}$ denotes the $\langle\cdot, \cdot\rangle_{h}$-orthogonal projection onto $\overline{\operatorname{Sp}}\left\{\xi_{n}: n>m\right\}$.

Lemma 4.8. Suppose $Q_{m} f=f$ for some $f \in L^{2}\left(S^{1}\right)$ and $m \geq 0$. If $\alpha_{m} \leq 1 /\left(2 \kappa^{2}\right)$ then $\|f\|_{h}^{2} \leq 2 \kappa^{2}\left\|\Pi^{+} f\right\|^{2}$.

Proof. Write $f=\sum_{n>m} \gamma_{n} \xi_{n}$ for some $\gamma_{n}$. Then

$$
\left\|\Pi^{-} f\right\|^{2} \leq \sum_{n>m}\left|\gamma_{n}\right|^{2} \sum_{n>m}\left\|\Pi^{-} \xi_{n}\right\|^{2}=\alpha_{m}\|f\|_{h}^{2} .
$$

Since $f=\Pi^{+} f+\Pi^{-} f$ is an orthogonal decomposition (47) now gives

$$
\|f\|_{h}^{2} \leq \kappa^{2}\|f\|^{2}=\kappa^{2}\left(\left\|\Pi^{+} f\right\|^{2}+\left\|\Pi^{-} f\right\|^{2}\right) \leq \kappa^{2}\left\|\Pi^{+} f\right\|^{2}+\frac{1}{2}\|f\|_{h}^{2} .
$$

The result follows.

Lemma 4.9. Suppose $M>0$ and $f \in \operatorname{Sp}\left\{\xi_{n}: 0<n \leq M\right\}$. Then

$$
\left\langle Q_{M} \Pi^{+} f, T Q_{M} \Pi^{+} f\right\rangle \leq M\left[\alpha_{0} \alpha_{M} \beta_{0} \beta_{M}\right]^{1 / 2}\|f\|_{h}^{2} .
$$

Proof. Write $f=\sum_{0<n \leq M} \gamma_{n} \xi_{n}$ for some $\gamma_{n}$. Then

$$
Q_{M} \Pi^{+} f=\sum_{n^{\prime}>M}\left\langle\xi_{n^{\prime}}, \Pi^{+} f\right\rangle_{h} \xi_{n^{\prime}}=\sum_{n^{\prime}>M} \sum_{0<n \leq M} \gamma_{n}\left\langle\xi_{n^{\prime}}, \Pi^{+} \xi_{n}\right\rangle_{h} \xi_{n^{\prime}} .
$$

Noting that $\left\langle\xi_{n^{\prime}}, \Pi^{+} \xi_{n}\right\rangle_{h}=-\left\langle\xi_{n^{\prime}}, \Pi^{-} \xi_{n}\right\rangle_{h}$ when $n \neq n^{\prime}$, (49) and (51) now give

$$
\begin{aligned}
\left\langle Q_{M} \Pi^{+} f, T Q_{M} \Pi^{+} f\right\rangle & \leq \sum_{n^{\prime}>M}\left(n^{\prime}-t\right)\left|\sum_{0<n \leq M} \gamma_{n}\left\langle\xi_{n^{\prime}}, \Pi^{-} \xi_{n}\right\rangle_{h}\right|^{2} \\
& \leq \sum_{n^{\prime}>M} n^{\prime}\left[\sum_{0<n \leq M}\left|\gamma_{n}\right|^{2} \sum_{0<n \leq M}\left|\left\langle\xi_{n^{\prime}}, \Pi^{-} \xi_{n}\right\rangle_{h}\right|^{2}\right] \\
& =\|f\|_{h}^{2} \sum_{0<n \leq M} \sum_{n^{\prime}>M} n^{\prime}\left|\left\langle\xi_{n^{\prime}}, \Pi^{-} \xi_{n}\right\rangle_{h}\right|^{2} .
\end{aligned}
$$


However, for any $n, n^{\prime}$ we have $\left\langle\xi_{n^{\prime}}, \Pi^{-} \xi_{n}\right\rangle_{h}=\left\langle\Pi^{-} h \xi_{n^{\prime}}, \Pi^{-} \xi_{n}\right\rangle$ while

$$
\begin{aligned}
n^{\prime} \overline{\left\langle\xi_{n^{\prime}}, \Pi^{-} \xi_{n}\right\rangle_{h}}=\left\langle\Pi^{-} \xi_{n}, n^{\prime} h \xi_{n^{\prime}}\right\rangle & =\left\langle\Pi^{-} \xi_{n},-i \nabla \xi_{n^{\prime}}\right\rangle \\
& =\left\langle\Pi^{-}(-i \nabla) \xi_{n}, \xi_{n^{\prime}}\right\rangle=n\left\langle\Pi^{-} h \xi_{n}, \Pi^{-} \xi_{n^{\prime}}\right\rangle .
\end{aligned}
$$

(note that, $\Pi^{-}$is an $\langle\cdot, \cdot\rangle$-orthogonal projection). Hence

$$
\begin{aligned}
\sum_{0<n \leq M} \sum_{n^{\prime}>M} n^{\prime}\left|\left\langle\xi_{n^{\prime}}, \Pi^{-} \xi_{n}\right\rangle_{h}\right|^{2} & =\sum_{0<n \leq M} \sum_{n^{\prime}>M} n\left\langle\Pi^{-} h \xi_{n^{\prime}}, \Pi^{-} \xi_{n}\right\rangle\left\langle\Pi^{-} h \xi_{n}, \Pi^{-} \xi_{n^{\prime}}\right\rangle \\
& \leq M \sum_{n>0}\left\|\Pi^{-} \xi_{n}\right\|\left\|\Pi^{-} h \xi_{n}\right\| \sum_{n^{\prime}>M}\left\|\Pi^{-} \xi_{n^{\prime}}\right\|\left\|\Pi^{-} h \xi_{n^{\prime}}\right\| \\
& \leq M\left[\alpha_{0} \beta_{0} \alpha_{M} \beta_{M}\right]^{1 / 2} .
\end{aligned}
$$

The result now follows from (52).

Proposition 4.10. Suppose $0 \leq m<M \leq t$. Let $X=\operatorname{Sp}\left\{\xi_{n}: m<n \leq M\right\}$ and $X^{+}=\Pi^{+} X \subset H^{2}\left(S^{1}\right)$. If $\alpha_{m} \leq 1 /\left(2 \kappa^{2}\right)$ then $\operatorname{dim} X^{+}=M-m$ and

$$
\langle f, T f\rangle \leq-\kappa^{2}\left(t-M-2 M\left[\alpha_{0} \alpha_{M} \beta_{0} \beta_{M}\right]^{1 / 2}\right)\|f\|^{2}, \quad f \in X^{+} .
$$

Proof. Let $f \in X$ and set $f^{+}=\Pi^{+} f \in X^{+}$. If $f^{+}=0$ then $f=0$ by Lemma 4.8; thus $\operatorname{dim} X^{+}=\operatorname{dim} X=M-m$. On the other hand, combining (47) with Lemmas $4.6,4.8$ and 4.9 gives

$$
\begin{aligned}
\left\langle f^{+}, T f^{+}\right\rangle & \leq(M-t)\left\|f^{+}\right\|_{h}^{2}+\left\langle Q_{M} f^{+}, T Q_{M} f^{+}\right\rangle \\
& \leq \kappa^{2}(M-t)\left\|f^{+}\right\|^{2}+M\left[\alpha_{0} \alpha_{M} \beta_{0} \beta_{M}\right]^{1 / 2}\|f\|_{h}^{2} \\
& \leq \kappa^{2}\left(M-t+2 M\left[\alpha_{0} \alpha_{M} \beta_{0} \beta_{M}\right]^{1 / 2}\right)\left\|f^{+}\right\|^{2}
\end{aligned}
$$

as required.

Proof of Proposition 4.3. Choose $m \geq 0$ so that $\alpha_{m} \leq 1 /\left(2 \kappa^{2}\right)$ (which is possible by Proposition 4.7). Also let

$$
\nu_{1, t}=\frac{1}{2 \kappa^{2}}\left(\nu_{t}+1\right)+2 C_{7}^{2} t^{(1-2 \alpha)_{+}}
$$

and $M_{t}=\min \left\{n \in \mathbb{N}_{0}: n \geq t-\nu_{1, t}-1\right\}$; in particular, $M_{t} \geq t-\nu_{1, t}-1$. Set

$$
X_{t}=\Pi^{+} \operatorname{Sp}\left\{\xi_{n}: m<n \leq M_{t}\right\} \subset H^{2}\left(S^{1}\right) .
$$

Proposition 4.10 gives $\operatorname{dim} X_{t} \geq M_{t}-m \geq t-\left(\nu_{1, t}+m+1\right)$ (note that, $X_{t}=\{0\}$ when $\left.M_{t} \leq m\right)$. The required estimate for $\operatorname{dim} X_{t}$ now follows if we take $C_{4,1}=$ $1 /\left(2 \kappa^{2}\right)$ and $C_{4,2}=C_{4,1}+2 C_{7}^{2}+m+1$ (note that, $t^{(1-2 \alpha)_{+}} \geq 1$ for $t \geq 1$ ).

Now let $0 \neq f \in X_{t}$. Then $1 \leq M_{t} \leq t-\nu_{1, t} \leq t$ (otherwise $X_{t}=\{0\}$ ), leading to $M_{t}\left(M_{t}+1\right)^{-2 \alpha} \leq M_{t}^{1-2 \alpha} \leq t^{(1-2 \alpha)_{+}}$. Propositions 4.10 and 4.7 then give

$$
\begin{aligned}
\langle f, T f\rangle & \leq \kappa^{2}\left(M_{t}-t+2 M_{t}\left[\alpha_{0} \alpha_{M_{t}} \beta_{0} \beta_{M_{t}}\right]^{1 / 2}\right)\|f\|^{2} \\
& \leq \kappa^{2}\left(M_{t}-t+2 C_{7}^{2} M_{t}\left(M_{t}+1\right)^{-2 \alpha}\right)\|f\|^{2} \\
& \leq \kappa^{2}\left(-\nu_{1, t}+2 C_{7}^{2} t^{(1-2 \alpha)_{+}}\right)\|f\|^{2}=-\frac{1}{2}\left(\nu_{t}+1\right)\|f\|^{2},
\end{aligned}
$$

so $\omega_{f} \leq-\nu_{t}$ by $(46)$. 


\section{Acknowledgements}

The author wishes to thank A. B. Pushnitski and I. Sorrell for several useful discussions. This research was supported by EPSRC under grant EP/E037410/1.

\section{References}

[1] R. A. Adams, Sobolev Spaces, Academic Press, New York (1975).

[2] Y. Aharonov and A. Casher,Ground state of a spin-1/2 charged particle in a two-dimensional magnetic field, Phys. Rev. A 19, no. 6 (1979) 2461-2462.

[3] Y. Colin de Verdiere, L'asymptotique de Weyl pour les bouteilles magnétiques, Commun. Math. Phys. 105 (1986) 327-335.

[4] H. D. Cornean, S. Fournais, R. Frank and B. Helffer, Sharp trace asymptotics for a class of 2D-magnetic operators, to appear in Ann. Henri Poincaré.

[5] P. G. Dodds and D. H. Fremlin, Compact Operators in Banach Lattices, Israel J. Math. 34 (1979) no. 4, 287-320.

[6] M. Dimassi and G. Raikov, Spectral asymptotics for quantum Hamiltonians in strong magnetic fields, Cubo Mat. Educ. 3 (2001) 317-391.

[7] L. Erdős, Ground state density of the Pauli operator in the large field limit, Lett. Math. Phys. 29, (1993) 219-240.

[8] L. Erdős and J. P. Solovej, Semiclassical eigenvalue estimates for the Pauli operator with strong non-homogeneous magnetic fields. II. Leading order asymptotic estimates, Commun. Math. Phys. 188 (1997) 599-656.

[9] L. Erdős and J. P. Solovej, Uniform Lieb-Thirring inequality for the three dimensional Pauli operator with a strong non-homogeneous magnetic field, Ann. Inst. H. Poincaré 5 (2004) 671-741.

[10] L. Erdős and V. Vougalter, Pauli Operator and Aharonov-Casher Theorem for Measure Valued Magnetic Fields, Commun. Math. Phys. 225 (2002) 399-421.

[11] S. Fournais and B. Helffer, Accurate eigenvalue estimates for the magnetic Neumann Laplacian, Ann. Inst. Fourier (Grenoble) 56 (2006) 1-67.

[12] R. L. Frank, On the asymptotic behavior of edge states for magnetic Schrödinger operators, Proc. Lond. Math. Soc. (3) 95 (2007), no. 1, 1-19.

[13] D. Gilbarg and N. S. Trudinger, Elliptic Partial Differential Equations of the Second Order, 2nd Edition, Corrected 3rd printing, Springer-Verlag, Berlin Heidelberg New York (1998).

[14] B. Helffer and A. Morame, Magnetic bottles in connection with superconductivity, J. Funct. Anal. 185 (2001) 604-680.

[15] D. Hundertmark and B. Simon, A diamagnetic inequality for semigroup differences, J. Reine Angew. Math. 571 (2004) 107-130. 
[16] F. Jones, Lebesgue Integration on Euclidean Space, Jones and Bartlett, Boston London (1993).

[17] T. Kato, Perturbation Theory for Linear Operators, 2nd Edition, SpringerVerlag, Berlin (1980).

[18] Y. Katznelson, An Introduction to Harmonic Analysis, 3rd Edition, Cambridge University Press, Cambridge (2004).

[19] H. Leinfelder, Gauge Invariance of Schrödinger Operators and Related Spectral Properties, J. Operator Theory 9 (1983) 163-179.

[20] E. Lieb, J.-P. Solovej and J. Yngvason, Asymptotics of heavy atoms in high magnetic fields. II. Semiclassical regions, Comm. Math. Phys. 161 (1994) 77124.

[21] L. D. Pitt, A Compactness Condition for Linear Operators on Function Spaces, J. Operator Theory 1 (1979) no. 1, 49-54.

[22] M. Reed and B. Simon, Methods of Modern Mathematical Physics II: SelfAdjointness, Academic Press, San Diego (1975).

[23] M. Reed and B. Simon, Methods of Modern Mathematical Physics IV: Analysis of Operators, Academic Press, San Diego (1979).

[24] G. Rozenblum, Semigroup Domination and Eigenvalue Estimates, Algebra $i$ Analiz 12 (2000) no. 5, 158-177; English transl., St. Petersburg Math. J. 12 (2001) no. 5, 831-845.

[25] A. V. Sobolev, Quasi-Classical Asymptotics for the Pauli Operator, Commun. Math. Phys. 194 (1998) 109-134.

[26] M. Soloymak, Spectral Problems Related to the Critical Exponent in the Sobolev Embedding Theorem, Proc. London Math. Soc. (3) 71 (1995) 53-75.

[27] H. Tamura, Asymptotic distribution of eigenvalues for Schrödinger operators with magnetic fields, Nagoya Math. J. 105 (1987) 49-69.

[28] F. Truc, Semi-classical asymptotics for magnetic bottles, Asymptotic Anal. 15 (1997) 385-395. 